\title{
Three-dimensional fracture analysis for a thin cracked hardening plate
}

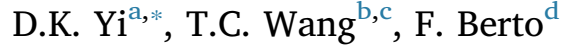 \\ ${ }^{a}$ College of Mechanics and Materials, Hohai University, China \\ ${ }^{\mathrm{b}}$ LNM, Institute of Mechanics, Chinese Academy of Science, China \\ ${ }^{\mathrm{c}}$ University of Chinese Academy of Sciences, China \\ ${ }^{\mathrm{d}}$ Mechanical and Industrial Engineering, Norwegian University of Science and Technology, Norway
}

\section{A R T I C L E I N F O}

\section{Keywords:}

Crack front stress fields

Elastic-plastic fracture

Three-dimensional numerical analysis

\begin{abstract}
A B S T R A C T
A semi-analytical method is presented to investigate fracture behavior of a mode-I crack in a thin ductile plate. In the part of theoretical analysis, a total deformation theory of plasticity, in conjunction with a power law hardening stress-strain relation, is employed. Three-dimensional Maxwell stress functions, the minimum complementary potential energy principle and three dimensional $J$-integrals are used to obtain crack front stress fields. In the part of numerical analysis, three dimensional finite element simulations are carried out to obtain $J$-integrals, the out-of-plane constraint level and crack front stress fields. Comparison with numerical results of crack front stress fields shows the analytic solutions for crack front stress fields are valid. Numerical and analytic results show that the value of the opening stress ahead of the crack front deceases with increasing $z$ (approaching the free surface) and lies between the corresponding plane strain and plane stress solutions.
\end{abstract}

\section{Introduction}

Plate elements bearing tension, compression or bending are widely applied in aerospace engineering and civil engineering. Stress analyses of cracked plate elements undoubtedly are important issues in fracture mechanics. In earlier studies, Rice and Rosengren [1] and Hutchinson [2] presented two-dimensional solutions for singular fields near the tip of a crack in a power law hardening material, known as HRR field solutions. Considering a three-dimensional thin elastic plate containing a through-thickness crack under pressure on the crack surfaces, Hartranft and Sih [3] and Sih [4] derived a closed form solution to depict the distribution of stress intensity factors in the plate thickness direction. The solution also revealed a boundary layer exists near the free surface of the plate. Nakamura and Parks investigated three-dimensional crack front fields in a thin elastic or plastic plate with a through-thickness crack under a remote $K_{\mathrm{I}}$ field using finite element methods. In the elastic case [5], these authors concluded that three-dimensional effects appear within a radial distance of about one-half thickness from the crack front on the mid-plane. In the plastic case [6], they concluded that for a low thin hardening plate under small scale yielding, the existence (at the mid-plane) of plane strain HRR-dominance requires the thickness of the plate to exceed $\frac{1}{2}\left(\frac{K_{I}}{\bar{\sigma}_{y}}\right)^{2}$. Here, $\bar{\sigma}_{y}$ is a yield stress. Su and Sun [7] carried out a three-dimensional asymptotic stress analysis for a cracked elastic plate under tension. They reported that the stress singularity at the crack front is of square root singularity throughout the thickness, while the stress intensity factor is a function of the thickness-wise coordinate. Using a Global-

\footnotetext{
* Corresponding author.

E-mail address: csruydk@163.com (D.K. Yi).
} 


\section{Nomenclature}

a normalized half crack length

$\bar{a} \quad$ half crack length

$A, B, C, D, E, F$ functions in Eq. (33)

$A\left(\Gamma_{2}\right) \quad$ domain bounded by $\Gamma_{2}$

$\widetilde{A}, \widetilde{B}, \widetilde{C}, \widetilde{D}, \widetilde{E}, \widetilde{F}, H$ functions in Eq. (35)

$\bar{E} \quad$ elastic modulus

$\bar{h} \quad$ half thickness of plate

$I \quad$ function relative to $J$

$K(z), K_{i}(z)$ amplitude of $\phi$ and $\phi_{i}, i=1,2,3$

$K_{\mathrm{I}} \quad$ mode-I stress intensity factor

$\sigma_{e} \quad$ equivalent stress

$\tilde{\sigma}_{e} \quad$ part of equivalent stress

$J(z), J(\eta)$ three-dimensional $J$-integral (in dimensionless quantity)

$J_{0} \quad J$-integral (in dimensionless quantity) caused by remote uniform tensile loading

$n \quad$ stain hardening coefficient

$n_{j} \quad$ components of a unit vector outward normal to integral path, $j=x, y$

$r, \theta, z \quad$ normalized cylindrical coordinates

$R \quad$ radius of cylindrical body

$r_{0} \quad$ normalized radius of $\Omega_{0}$

$r_{1}, r_{2} \quad$ normalized radius of integral path

$r_{p} \quad$ plastic zone size

$\bar{r}, \theta, \bar{z} \quad$ cylindrical coordinates

$g, s, s_{1}, s_{2}, s_{3}$ exponents of $r$

$s_{i j} \quad$ components of deviatoric stress normalized by $\bar{\sigma}_{y}$

$T \quad$ out-of-plane constraint level

$u_{i} \quad$ components of normalized displacement, $i=x, y$, $z$ in rectangular coordinates or $i=r, \theta, z$ in cylindrical coordinate

$\tilde{u}_{i} \quad$ part of $u_{i}$

$x, y, z \quad$ normalized rectangular coordinates

$\bar{x}, \bar{y}, \bar{z} \quad$ rectangular coordinates

$W \quad$ strain energy density (in dimensionless quantity)

$\Gamma_{1}, \Gamma_{2} \quad$ integral path with radius $r_{1}, r_{2}$

$\varepsilon \quad$ strain tensor

$\varepsilon_{i j} \quad$ components of strain normalized by $\bar{\varepsilon}_{y}, i$ or $j=x$, $y, z$ in rectangular coordinates, $i$ or $j=r, \theta, z$ in cylindrical coordinates

$\tilde{\varepsilon}_{i j} \quad$ part of $\varepsilon_{i j}$

$\bar{\varepsilon}_{y} \quad$ yield strain

$v \quad$ Poisson's ratio

$\rho \quad$ one of spherical coordinates

$\sigma \quad$ stress tensor

$\sigma_{i j} \quad$ components of normalized stress, $i$ or $j=x, y, z$ in rectangular coordinates, $i$ or $j=r, \theta, z$ in cylindrical coordinates

$\sigma^{\infty} \quad$ normalized remote uniform tensile loading

$\bar{\sigma}^{\infty} \quad$ remote uniform tensile loading

$\bar{\sigma}_{i j} \quad$ components of stress, $i$ or $j=x, y, z$ in rectangular coordinates, $i$ or $j=r, \theta, z$ in cylindrical coordinates

$\bar{\sigma}_{y} \quad$ yield stress

$\phi, \phi_{i} \quad$ normalized Maxwell stress function, $i=1,2,3$

$\bar{\phi}_{i} \quad$ Maxwell stress function, $i=1,2,3$

$\widetilde{\phi} \quad$ part of $\phi$

$\Omega_{0} \quad$ a cylindrical body including the crack front

local finite element technique, Kwon and Sun [8] performed three-dimensional finite element analyses for an elastic plate with a through-thickness crack under remote loading. Based on their numerical results, Kwon and Sun proposed a $K$ - $V$ interaction model to reveal the characterization of the three-dimensional crack front stress fields in the boundary layer (the region close to the plate free surface). Based on the solution for an edge dislocation in infinite plates of arbitrary thickness and an application of the distributed dislocation technique, Kotousov [9] investigated the effect of plate thickness on the stress intensity factor. The author believed that the stress intensity factor matches the classical plane solution in the case of short cracks or very thick plates, and the stress intensity factor is related to the classical plane solution by $\sqrt{1-v^{2}}$ in the case of long cracks. Based on the strip-yield assumption and the application of the distributed dislocation technique, Codrington and Kotousov [10] presented a new analytical method for calculating plasticity-induced fatigue crack closure in plates of finite thickness under cyclic loading. The authors showed the effect of plate thickness on the plastic zone size and concluded that the reverse to direct plastic zone size ratio decreases with a decrease in the plate thickness to crack length ratio. Using a novel eigenfunction expansion technique, Chaudhuri [11] derived a three-dimensional asymptotic crack front fields for an infinite cracked elastic plate made of a homogeneous cubic single crystal under remote mode-I/II loading. The author concluded that no crack deviation is expected when single crystals of alkali halides (rock salt structure) are subjected to tensile loading, but crack deflection always occurs when single crystals of bcc metals are subjected to tensile loading. Employing finite element methods, Sobotka and Dodds [12] investigated key features of elastic-plastic fields near a steadily advancing three-dimensional crack in a thin Al-Ti alloy plate under small-scale yielding conditions. Their numerical results showed that three-dimensional effects on the crack front stress fields begin to appear at distances of about 1.5-2.5 times the plate thickness ahead of the advancing front. Khan et al. [13] carried out a photoelastic experiment to investigate the effect of plate thickness on the plastic zone size in a plate with a through-thickness crack under mixed mode loading. These authors observed that the plastic zone size decreases with increasing thickness of the plate both in the mid-plane and at the surface. Xiang and Guo [14] proposed a set of empirical formulae to depict the three-dimensional stress fields near the front of a through-thickness crack in a ductile plate under tensile loading. Kotousov et al. [15] investigated the effect of plate thickness on coupled stress intensity factors for an elastic plate with a through-thickness crack under shear or anti-plane loading. These authors argued that shear or anti-plane loading always generates coupled local fracture modes (mode-II + mode-III) due to Poisson's ratio effect and the coupled stress intensity factors can be expressed by a power function of plate thickness. Considering a plate with a through-thickness crack under remote loading, Wang et al. $[16,17]$ investigated the effects of in-plane and out of-plane dimensions on crack front stress fields, $J$-integrals, CTOD and cracktip constraint parameters using three-dimensional finite element technologies. These authors believed that the distribution of the 
local $J$-integral or the out-of-plane constraint level along the crack front becomes more uniform with increasing plate thickness. Using single-edge-notched bend and compact specimens as well as three-dimensional finite element methods, Shlyannikov et al. [18] carried out experimental and numerical studies on the fracture toughness of ductile plates. Experimental and numerical results obtained by these authors demonstrated that the plastic stress intensity factor which is sensitive to in-plane and out-of-plane constraint levels can be used to characterize the fracture resistance characteristics as a unified single parameter for a variety of specimen geometries. Using three dimensional finite element methods, Nikishkov and Matvienko [19] investigated the distributions of $J$ integral and the constraint parameter $A$ along the front of a mode-I crack in a power-law hardening plate. Here, the constraint parameter $A$ is an amplitude of the second and third terms in an elastic-plastic asymptotic expansion for the crack tip stress field and is considered as a measure of stress field deviation from the HRR field solutions. These authors concluded that the constraint parameter $A$ significantly decreases with increasing specimen thickness. Kotousov et al. [20] applied the first order plate theory to analytically obtain the transverse displacement at the tip of a semi-infinite crack loaded in mode-I.

In the papers mentioned above, there are a number of numerical results on three-dimensional elastic or plastic fracture problems. However, there are a very limited number of analytical solutions available. In the current paper, we focused on obtaining semianalytical solutions for stress fields near the front of a crack in a ductile plate. In Section 2, physical backgrounds, basic equations and hypotheses for the current problem are described. In Section 3, solutions for crack front stress fields and the plastic zone size ahead of the crack front are presented. Three-dimensional finite element simulations for $J(z), T(z)$, the distribution of the opening stresses in the thickness direction and the angular-variations of in-plane stresses on the mid-plane are carried out. In Section 4, analytic and

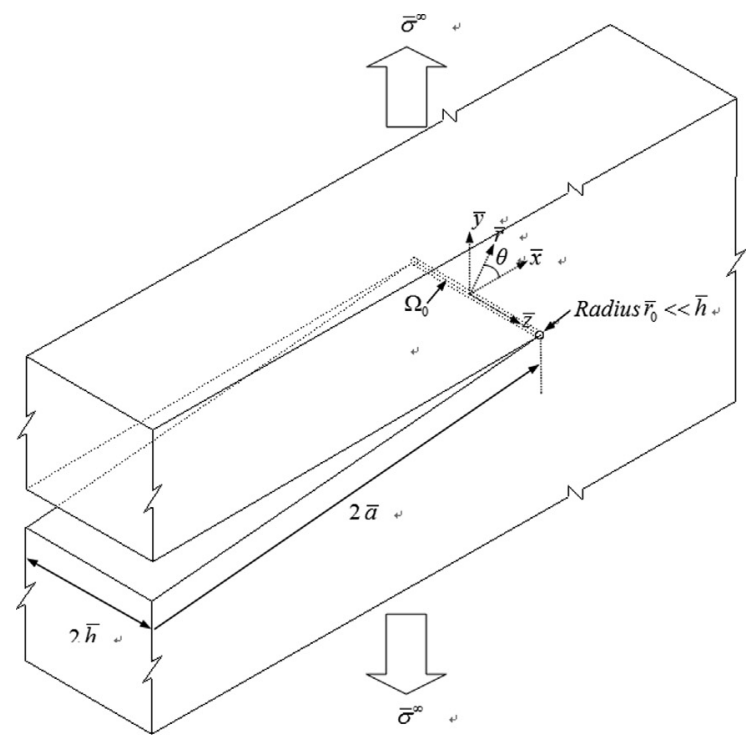

(a)

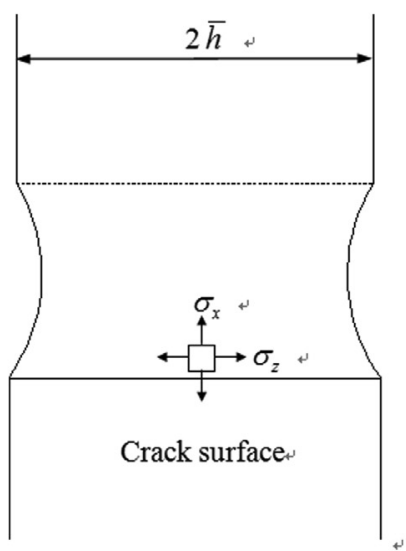

(b)

Fig. 1. A thin plate containing a through-the-thickness crack subjected to remote tensile loading $\bar{\sigma}^{\infty}$. (a) A cracked thin plate under tension and (b) a triaxial stress state near the crack front. 
numerical results are discussed and compared. In Section 5, a brief summary and some conclusions are given.

\section{Basic equations and expressions of crack front fields}

\subsection{Basic equations}

Consider a thin hardening plate containing a through-thickness crack subjected to remote tensile loading $\bar{\sigma}^{\infty}$. The thickness of the plate is $2 \bar{h}$, illustrated in Fig. 1a. Disregarding elastic deformations, the tensile stress-strain relations of power hardening materials may be presented as

$$
\varepsilon=\alpha \sigma^{n} \text {. }
$$

Throughout this paper, unless otherwise stated, all unbarred quantities are non-dimensional quantities. Length quantities if unbarred will be non-dimensionalized by the half thickness of the plate $\bar{h}$. Stress quantities if unbarred will be non-dimensionalized by a yield stress $\bar{\sigma}_{y}$ and unbarred quantities $\varepsilon$ will be normalized with respect to the corresponding yield strain $\bar{\varepsilon}_{y}=\bar{\sigma}_{y} / \bar{E}$ where $\bar{E}$ is the elastic modulus. $\alpha$ is a constant and $n$ is the stain hardening coefficient.

According to the total deformation theory, the generalized stress-strain relation which reduces to Eq. (1) in simple tension may be written as

$$
\varepsilon_{i j}=\frac{3}{2} \alpha \sigma_{e}^{n-1} s_{i j}=\frac{3}{2} \alpha \sigma_{e}^{n-1}\left(\sigma_{i j}-\frac{1}{3} \sigma_{k k} \delta_{i j}\right) .
$$

Here,

$$
\sigma_{e}=\sqrt{\frac{3}{2} s_{i j} s_{i j}}
$$

Equilibrium equations disregarding body forces may be written as

$$
\bar{\sigma}_{i j, j}=0 \text {. }
$$

When Maxwell stress functions are introduced, the stress components satisfying Eq. (4) may be written as

$$
\left.\begin{array}{c}
\sigma_{x}=\frac{\partial^{2} \phi_{2}}{\partial z^{2}}+\frac{\partial^{2} \phi_{3}}{\partial y^{2}}, \sigma_{y}=\frac{\partial^{2} \phi_{1}}{\partial z^{2}}+\frac{\partial^{2} \phi_{3}}{\partial x^{2}}, \sigma_{z}=\frac{\partial^{2} \phi_{1}}{\partial y^{2}}+\frac{\partial^{2} \phi_{2}}{\partial x^{2}}, \\
\sigma_{x y}=-\frac{\partial^{2} \phi_{3}}{\partial x \partial y}, \sigma_{x z}=-\frac{\partial^{2} \phi_{2}}{\partial x \partial z}, \sigma_{y z}=-\frac{\partial^{2} \phi_{1}}{\partial y \partial z} .
\end{array}\right\}
$$

Considering a cylindrical coordinate system, shown in Fig. 1a, one may have the following relationships

$$
\left.\begin{array}{c}
\frac{\partial^{2}}{\partial x^{2}}=\cos ^{2} \theta \frac{\partial}{\partial r^{2}}-\frac{\sin 2 \theta}{r} \frac{\partial^{2}}{\partial r \partial \theta}+\frac{\sin ^{2} \theta}{r} \frac{\partial}{\partial r}+\frac{\sin 2 \theta}{r^{2}} \frac{\partial}{\partial \theta}+\frac{\sin ^{2} \theta}{r^{2}} \frac{\partial}{\partial \theta^{2}}, \\
\frac{\partial^{2}}{\partial y^{2}}=\sin ^{2} \theta \frac{\partial}{\partial r^{2}}+\frac{\sin 2 \theta}{r} \frac{\partial^{2}}{\partial r \partial \theta}+\frac{\cos ^{2} \theta}{r} \frac{\partial}{\partial r}-\frac{\sin 2 \theta}{r^{2}} \frac{\partial}{\partial \theta}+\frac{\cos ^{2} \theta}{r^{2}} \frac{\partial}{\partial \theta^{2}}, \\
\frac{\partial^{2}}{\partial x \partial y}=\sin \theta \cos \theta \frac{\partial}{\partial r^{2}}+\frac{\cos 2 \theta}{r} \frac{\partial^{2}}{\partial r \partial \theta}-\frac{\sin 2 \theta}{2 r} \frac{\partial}{\partial r}-\frac{\cos 2 \theta}{r^{2}} \frac{\partial}{\partial \theta}-\frac{\sin 2 \theta}{2 r^{2}} \frac{\partial}{\partial \theta^{2}}, \\
\frac{\partial^{2}}{\partial x \partial z}=\cos \theta \frac{\partial^{2}}{\partial r \partial z}-\frac{\sin \theta}{r} \frac{\partial^{2}}{\partial \theta \partial z}, \frac{\partial^{2}}{\partial y \partial z}=\sin \theta \frac{\partial^{2}}{\partial r \partial z}+\frac{\cos \theta}{r} \frac{\partial^{2}}{\partial \theta \partial z} .
\end{array}\right\}
$$

Substituting Eq. (6) to Eq. (5), the stress components may be re-written as

$$
\left.\begin{array}{c}
\sigma_{x}=\frac{\partial^{2} \phi_{2}}{\partial z^{2}}+\left(\sin ^{2} \theta \frac{\partial}{\partial r^{2}}+\frac{\sin 2 \theta}{r} \frac{\partial^{2}}{\partial r \partial \theta}+\frac{\cos ^{2} \theta}{r} \frac{\partial}{\partial r}-\frac{\sin 2 \theta}{r^{2}} \frac{\partial}{\partial \theta}+\frac{\cos ^{2} \theta}{r^{2}} \frac{\partial}{\partial \theta^{2}}\right) \phi_{3}, \\
\sigma_{y}=\frac{\partial^{2} \phi_{1}}{\partial z^{2}}+\left(\cos ^{2} \theta \frac{\partial}{\partial r^{2}}-\frac{\sin 2 \theta}{r} \frac{\partial^{2}}{\partial r \partial \theta}+\frac{\sin ^{2} \theta}{r} \frac{\partial}{\partial r}+\frac{\sin 2 \theta}{r^{2}} \frac{\partial}{\partial \theta}+\frac{\sin ^{2} \theta}{r^{2}} \frac{\partial}{\partial \theta^{2}}\right) \phi_{3}, \\
\sigma_{z}=\left(\sin ^{2} \theta \frac{\partial}{\partial r^{2}}+\frac{\sin 2 \theta}{r} \frac{\partial^{2}}{\partial r \partial \theta}+\frac{\cos ^{2} \theta}{r} \frac{\partial}{\partial r}-\frac{\sin 2 \theta}{r^{2}} \frac{\partial}{\partial \theta}+\frac{\cos ^{2} \theta}{r^{2}} \frac{\partial}{\partial \theta^{2}}\right) \phi_{1} \\
+\left(\cos ^{2} \theta \frac{\partial}{\partial r^{2}}-\frac{\sin 2 \theta}{r} \frac{\partial^{2}}{\partial r \partial \theta}+\frac{\sin ^{2} \theta}{r} \frac{\partial}{\partial r}+\frac{\sin 2 \theta}{r^{2}} \frac{\partial}{\partial \theta}+\frac{\sin ^{2} \theta}{r^{2}} \frac{\partial}{\partial \theta^{2}}\right) \phi_{2}, \\
\sigma_{x y}=-\left(\frac{\sin 2 \theta}{2} \frac{\partial}{\partial r^{2}}+\frac{\cos 2 \theta}{r} \frac{\partial^{2}}{\partial r \partial \theta}-\frac{\sin 2 \theta}{2 r} \frac{\partial}{\partial r}-\frac{\cos 2 \theta}{r^{2}} \frac{\partial}{\partial \theta}-\frac{\sin 2 \theta}{2 r^{2}} \frac{\partial}{\partial \theta^{2}}\right) \phi_{3}, \\
\sigma_{x z}=-\left(\cos \theta \frac{\partial^{2}}{\partial r \partial z}-\frac{\sin \theta}{r} \frac{\partial^{2}}{\partial \theta \partial z}\right) \phi_{2}, \sigma_{y z}=-\left(\sin \theta \frac{\partial^{2}}{\partial r \partial z}+\frac{\cos \theta}{r} \frac{\partial^{2}}{\partial \theta \partial z}\right) \phi_{1} .
\end{array}\right\}
$$

The relationship between stress components in cylindrical coordinates and those in rectangular coordinates may be expressed as

$$
\left.\begin{array}{l}
\sigma_{r}=\sigma_{x} \cos ^{2} \theta+\sigma_{y} \sin ^{2} \theta+\sin 2 \theta \sigma_{x y}, \sigma_{\theta}=\sigma_{x} \sin ^{2} \theta+\sigma_{y} \cos ^{2} \theta-\sin 2 \theta \sigma_{x y}, \sigma_{z}=\sigma_{z}, \\
\sigma_{r \theta}=\sigma_{x y} \cos 2 \theta-\frac{\sigma_{x}-\sigma_{y}}{2} \sin 2 \theta, \sigma_{r z}=\sin \theta \sigma_{y z}+\cos \theta \sigma_{x z}, \sigma_{\theta z}=\cos \theta \sigma_{y z}-\sin \theta \sigma_{z x} .
\end{array}\right\}
$$

Substituting Eq. (7) into Eq. (8), one may obtain 


$$
\begin{aligned}
& \sigma_{r}=\cos ^{2} \theta \frac{\partial^{2} \phi_{2}}{\partial z^{2}}+\sin ^{2} \theta^{\partial^{2} \phi_{1}} \frac{1}{\partial z^{2}}+\left(\frac{1}{r}\left(\phi_{3}\right)^{\prime}+\frac{1}{r^{2}}\left(\phi_{3}\right)^{\cdot *}\right), \\
& \sigma_{\theta}=\sin ^{2} \theta \frac{\partial^{2} \phi_{2}}{\partial z^{2}}+\cos ^{2} \theta \frac{\partial^{2} \phi_{1}}{\partial z^{2}}+\left(\phi_{3}\right)^{\prime \prime}, \\
& \sigma_{z}=\sin ^{2} \theta\left(\phi_{1}\right)^{\prime \prime}+\frac{\sin 2 \theta}{r}\left(\phi_{1}\right)^{*^{\prime}}+\frac{\cos ^{2} \theta}{r}\left(\phi_{1}\right)^{\prime}-\frac{\sin 2 \theta}{r^{2}}\left(\phi_{1}\right)^{\cdot}+\frac{\cos ^{2} \theta}{r^{2}}\left(\phi_{1}\right)^{\cdot}+ \\
& \cos ^{2} \theta\left(\phi_{2}\right)^{\prime \prime}-\frac{\sin 2 \theta}{r}\left(\phi_{2}\right)^{0^{\prime}}+\frac{\sin ^{2} \theta}{r}\left(\phi_{2}\right)^{\prime}+\frac{\sin 2 \theta}{r^{2}}\left(\phi_{2}\right)^{\bullet}+\frac{\sin ^{2} \theta}{r^{2}}\left(\phi_{2}\right)^{\cdot \cdot}, \\
& \sigma_{r \theta}=-\frac{\sin 2 \theta}{2}\left(\frac{\partial^{2} \phi_{2}}{\partial z^{2}}-\frac{\partial^{2} \phi_{1}}{\partial z^{2}}\right)+\frac{1}{r^{2}}\left(\phi_{3}\right)^{\cdot}-\frac{1}{r}\left(\phi_{3}\right)^{0^{\prime}} \text {, } \\
& \sigma_{r z}=-\sin \theta\left(\sin \theta \frac{\partial\left(\phi_{1}\right)^{\prime}}{\partial z}+\frac{\cos \theta}{r} \frac{\partial\left(\phi_{1}\right)^{*}}{\partial z}\right)-\cos \theta\left(\cos \theta \frac{\partial\left(\phi_{2}\right)^{\prime}}{\partial z}-\frac{\sin \theta}{r} \frac{\partial\left(\phi_{2}\right)^{*}}{\partial z}\right), \\
& \sigma_{\theta z}=-\cos \theta\left(\sin \theta \frac{\partial\left(\phi_{1}\right)^{\prime}}{\partial z}+\frac{\cos \theta}{r} \frac{\partial\left(\phi_{1}\right)^{*}}{\partial z}\right)+\sin \theta\left(\cos \theta \frac{\partial\left(\phi_{2}\right)^{\prime}}{\partial z}-\frac{\sin \theta}{r} \frac{\partial\left(\phi_{2}\right)^{*}}{\partial z}\right) .
\end{aligned}
$$

Here, ()$^{*}=\frac{\partial}{\partial \theta}$ and ()$^{\prime}=\frac{\partial}{\partial r}$. The non-dimensional Maxwell stress functions $\phi_{i}, r$ and $z$ are given in terms of the dimensional quantities (barred) by

$$
\phi_{i}=\frac{\bar{\phi}_{i}}{\bar{\sigma}_{y} \bar{h}^{2}}, \quad r=\frac{\bar{r}}{\bar{h}}, \quad z=\frac{\bar{z}}{\bar{h}} .
$$

It can be proved that Eq. (9) satisfies the equilibrium equations in cylindrical coordinates, expressed as

$$
\left.\begin{array}{c}
\left(\sigma_{r}\right)^{\prime}+\frac{1}{r}\left(\sigma_{r \theta}\right)^{\cdot}+\frac{\partial \sigma_{r z}}{\partial z}+\frac{\sigma_{r}-\sigma_{\theta}}{r}=0, \\
\left(\sigma_{r \theta}\right)^{\prime}+\frac{1}{r}\left(\sigma_{\theta}\right)^{\cdot}+\frac{\partial \sigma_{\theta z}}{\partial z}+\frac{2 \sigma_{r \theta}}{r}=0, \\
\left(\sigma_{r z}\right)^{\prime}+\frac{1}{r}\left(\sigma_{\theta z}\right)^{\cdot}+\frac{\partial \sigma_{z}}{\partial z}+\frac{\sigma_{r z}}{r}=0 .
\end{array}\right\}
$$

\subsection{Expressions for three-dimensional crack front fields}

It is well known that a thin plate without a crack under tension is in a state of plane stress. However, When a thin plate containing a through-thickness crack is subjected to tensile loading, materials near the crack front should contract in the $\bar{x}$ and $\bar{z}$ directions due to the high stresses normal to the crack plane, but these materials are prevented from doing so by the surrounding materials. The outof-plane and in-plane constraints cause a three-dimensional stress state near the crack front, as Fig. 1b shows.

Consider a cylindrical body $\Omega_{0}$ including the crack front (see Fig. 1a) where $r_{0}<<h$. In the mid-plane, the out-of-plane constrain level is high, and at the free surface, the out-of-plane stress vanishes. According to the description above, the stress functions are attempted in the form

$$
\phi_{3}=\phi=K(z) r^{s} \tilde{\phi}(\theta, z)
$$

and

$$
\phi_{1}=\phi_{2}=T(z) \phi=T K r^{s} \tilde{\phi}
$$

Here, $T$ represents the out-of-plane constraint level varying in the thickness direction.

Substituting Eq. (12) and Eq. (13) to Eq. (9) and disregarding terms containing $r^{s}$ and $r^{s-1}$, one may obtain

$$
\left.\begin{array}{c}
\sigma_{r}=\frac{\phi^{\prime}}{r}+\frac{\phi^{\cdot}}{r^{2}}=K r^{s-2}\left(s \tilde{\phi}+\tilde{\phi}^{\cdot *}\right)=K r^{s-2} \widetilde{\sigma}_{r}, \\
\sigma_{\theta}=\phi^{\prime \prime}=K r^{s-2} s(s-1) \tilde{\phi}=K r^{s-2} \widetilde{\sigma}_{\theta}, \\
\sigma_{z}=\phi^{\prime \prime}+\frac{\phi^{\prime}}{r}+\frac{\phi^{\cdot}}{r^{2}}=T K r^{s-2}\left(s^{2} \widetilde{\phi}+\widetilde{\phi}^{\cdot \cdot}\right)=T K r^{s-2} \widetilde{\sigma}_{z}, \\
\sigma_{r \theta}=\frac{\phi^{\cdot}}{r^{2}}-\frac{\phi^{\prime}}{r}=K r^{s-2}(1-s) \tilde{\phi}^{\cdot}=K r^{s-2} \widetilde{\sigma}_{r \theta}, \\
\sigma_{r z}=\sigma_{\theta z}=0 .
\end{array}\right\}
$$

Here, one may find

$$
T=\frac{\sigma_{z}}{\sigma_{r}+\sigma_{\theta}}
$$

Substituting Eq. (14) to Eq. (2), the strain components near the crack front may be written as 


$$
\begin{aligned}
& \varepsilon_{r}=\frac{\alpha}{2} \sigma_{e}^{n-1}\left((2-T)\left(\frac{\phi^{\prime}}{r}+\frac{\phi^{*}}{r^{2}}\right)-(1+T) \phi^{\prime}\right) \\
& =\frac{\alpha}{2} K^{n} r^{(s-2) n} \widetilde{\sigma}_{e}^{n-1}\left(2\left(s \tilde{\phi}+\tilde{\phi}^{\cdot *}\right)-s(s-1) \tilde{\phi}-T\left(s^{2} \tilde{\phi}+\widetilde{\phi} \cdot *\right)\right)=\frac{\alpha}{2} K^{n} r^{(s-2) n} \widetilde{\varepsilon}_{r}, \\
& \varepsilon_{\theta}=\frac{\alpha}{2} \sigma_{e}^{n-1}\left((2-T) \phi^{\prime \prime}-(1+T)\left(\frac{\phi^{\prime}}{r}+\frac{\phi^{*}}{r^{2}}\right)\right) \\
& \left.=\frac{\alpha}{2} K^{n} r^{(s-2) n} \widetilde{\sigma}_{e}^{n-1}\left(2 s(s-1) \tilde{\phi}-\left(s \tilde{\phi}+\widetilde{\phi}^{*}\right)-T\left(s^{2} \tilde{\phi}+\widetilde{\phi}^{*}\right)\right)=\frac{\alpha}{2} K^{n} r^{(s-2) n} \widetilde{\varepsilon}_{\theta},\right\} \\
& \varepsilon_{z}=\alpha \sigma_{e}^{n-1} \frac{2 T-1}{2}\left(\frac{\dot{\phi}}{r}+\frac{\phi^{\cdot \cdot}}{r^{2}}+\phi^{\prime}\right) \\
& =\frac{\alpha}{2} K^{n} r^{(s-2) n} \widetilde{\sigma}_{e}^{n-1}(2 T-1)\left(s^{2} \tilde{\phi}+\widetilde{\phi}^{\cdot}\right)=\frac{\alpha}{2} K^{n} r^{(s-2) n} \widetilde{\varepsilon}_{z}, \\
& \varepsilon_{r \theta}=-\frac{3}{2} \alpha \sigma_{e}^{n-1}\left(\frac{\phi^{\prime}}{r}-\frac{\phi^{*}}{r^{2}}\right)=-\frac{3 \alpha}{2} K^{n} r^{(s-2) n} \widetilde{\sigma}_{e}^{n-1}(s-1) \tilde{\phi}^{\cdot}=\frac{\alpha}{2} K^{n} r^{s-2} \widetilde{\varepsilon}_{r \theta}, \\
& \varepsilon_{r z}=0, \varepsilon_{\theta z}=0 .
\end{aligned}
$$

Here,

$$
\begin{gathered}
\sigma_{e}=\sqrt{\frac{3}{2}\left[\left(\sigma_{r}-\frac{\sigma_{r}+\sigma_{\theta}+\sigma_{z}}{3}\right)^{2}+\left(\sigma_{\theta}-\frac{\sigma_{r}+\sigma_{\theta}+\sigma_{z}}{3}\right)^{2}\right.} \\
\left.+\left(\sigma_{z}-\frac{\sigma_{r}+\sigma_{\theta}+\sigma_{z}}{3}\right)^{2}+2\left(\sigma_{r \theta}\right)^{2}\right] \\
=K r^{s-2} \sqrt{\frac{3}{2}\left[\left(\widetilde{\sigma}_{\theta}-\frac{1+T}{3}\left(\widetilde{\sigma}_{\theta}+\widetilde{\sigma}_{r}\right)\right)^{2}+\left(\widetilde{\sigma}_{r}-\frac{1+T}{3}\left(\widetilde{\sigma}_{\theta}+\widetilde{\sigma}_{r}\right)\right)^{2}\right.} \\
\left.+\left(\widetilde{\sigma}_{z}-\frac{1+T}{3}\left(\widetilde{\sigma}_{\theta}+\widetilde{\sigma}_{r}\right)\right)^{2}+2\left(\widetilde{\sigma}_{r \theta}\right)^{2}\right] \\
=K r^{s-2} \widetilde{\sigma}_{e}
\end{gathered}
$$

and

$$
\begin{gathered}
\widetilde{\sigma}_{e}=\sqrt{\frac{3}{2}\left[\left(s(s-1) \tilde{\phi}-\frac{1+T}{3}\left(\tilde{\phi}^{\cdot}+s^{2} \tilde{\phi}\right)\right)^{2}+\left(s \tilde{\phi}+\widetilde{\phi}^{\cdot}-\frac{1+T}{3}\left(\tilde{\phi}^{\cdot}+s^{2} \widetilde{\phi}\right)\right)^{2}\right.} \\
\left.+\left(\frac{2 T-1}{3}\left(\tilde{\phi}^{\cdot}+s^{2} \widetilde{\phi}\right)\right)^{2}+2(1-s)^{2}\left(\tilde{\phi}^{\cdot}\right)^{2}\right]
\end{gathered}
$$

According to the principle of minimum complementary potential energy, one may have

$$
\iiint_{V}\left(\varepsilon_{x} \delta \sigma_{x}+\varepsilon_{y} \delta \sigma_{y}+\varepsilon_{z} \delta \sigma_{z}+\gamma_{x y} \delta \sigma_{x y}+\gamma_{x z} \delta \sigma_{x z}+\gamma_{y z} \delta \sigma_{y z}\right) d x d y d z=0
$$

Substituting Eq. (5) into Eq. (19) and employing variational methods, one may obtain

$$
\left.\begin{array}{c}
\iiint_{\mathrm{V}}\left(\varepsilon_{x} \delta \sigma_{x}+\varepsilon_{y} \delta \sigma_{y}+\varepsilon_{z} \delta \sigma_{z}+\gamma_{x y} \delta \sigma_{x y}\right) d x d y d z \\
=\iiint_{\mathrm{V}}\left(\left(\varepsilon_{x} \delta \frac{\partial^{2} \phi}{\partial y^{2}}+\varepsilon_{y} \delta \frac{\partial^{2} \phi}{\partial x^{2}}-\gamma_{x y} \delta \frac{\partial^{2} \phi}{\partial x \partial y}\right)+\varepsilon_{z} \delta\left(T\left(\frac{\partial^{2} \phi}{\partial y^{2}}+\frac{\partial^{2} \phi}{\partial x^{2}}\right)\right)\right) d V \\
=\iiint_{\mathrm{V}}\left(\left(\frac{\partial^{2} \varepsilon_{x}}{\partial y^{2}}+\frac{\partial^{2} \varepsilon_{y}}{\partial x^{2}}-\frac{\partial^{2} \gamma_{x y}}{\partial x \partial y}\right)+T\left(\frac{\partial^{2} \varepsilon_{z}}{\partial y^{2}}+\frac{\partial^{2} \varepsilon_{z}}{\partial x^{2}}\right)\right) \delta \phi d V=0 .
\end{array}\right\}
$$

Here, $\gamma_{x z}$ and $\gamma_{y z}$ are disregarded.

Considering $\delta \phi$ is arbitrary, one may obtain the equation which strain components near the crack front should meet, shown as

$$
\left(\frac{\partial^{2} \varepsilon_{x}}{\partial y^{2}}+\frac{\partial^{2} \varepsilon_{y}}{\partial x^{2}}-\frac{\partial^{2} \gamma_{x y}}{\partial x \partial y}\right)+T\left(\frac{\partial^{2} \varepsilon_{z}}{\partial x^{2}}+\frac{\partial^{2} \varepsilon_{z}}{\partial y^{2}}\right)=0
$$

The expression of Eq. (21) in terms of cylindrical coordinates may be written as

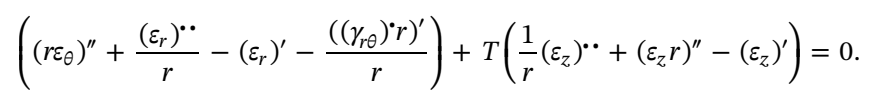

\section{Solutions for three-dimensional crack front fields}

\subsection{Solution for $s$}

In order to obtain $s$, a three-dimensional $J$-integral (in dimensionless quantity) may be introduced. A mathematical expression for 
the three-dimensional $J$-integral $[21-27]$ is

$$
\begin{gathered}
J(\eta)=\int_{\Gamma_{1}}\left(W d y-\sigma_{i j} n_{j} u_{i, x} d s\right),\left(r_{1} \rightarrow 0\right) \\
=\int_{\Gamma_{2}}\left(W d y-\sigma_{i j} n_{j} u_{i, x} d s\right)-\int_{A\left(\Gamma_{2}\right)} \frac{\partial\left(\sigma_{i z} u_{i, x}\right)}{\partial z} d A\left(\Gamma_{2}\right), i=x, y, z, j=x, y .
\end{gathered}
$$

Here, $\eta$ is a point along the crack front, $J(\eta)$ is the point-wise value of the $J$-integral at a given point $\eta$ along the crack front (see Fig. 2), $W$ is the strain energy density (in dimensionless quantity) for a linear or non-linear material, $\sigma_{i j}$ and $u_{i}$ are the Cartesian components of stress and displacement. Integral paths $\Gamma_{1}$ (radius $r_{1}$ ) and $\Gamma_{2}$ (radius $r_{2}$ ) lie in the plane perpendicular to the crack front, and $n_{j}$ are the components of a unit vector outward normal to the integral paths and normal to the crack front. $A\left(\Gamma_{2}\right)$ is the domain bounded by $\Gamma_{2}$.

The strain energy density $W$ can be expressed as [2]

$$
W=\int_{0}^{\varepsilon_{i j}} \sigma_{i j} d \varepsilon_{i j}=\alpha n \int_{0}^{\sigma_{e}} \sigma_{e}^{n} d \sigma_{e}=\frac{\alpha n}{n+1} K^{n+1} r^{(s-2)(n+1)} \tilde{\sigma}_{e}^{n+1} .
$$

Near the crack front, $\sigma_{z x}$ and $\sigma_{z y}$ may be disregarded, then we have [2]

$$
\begin{gathered}
\sigma_{i j} n_{j} u_{i, x}=\sigma_{x} n_{x} u_{x, x}+\sigma_{x y} n_{y} u_{x, x}+\sigma_{y x} n_{x} u_{y, x}+\sigma_{y} n_{y} u_{y, x} \\
=\alpha K^{n+1} r^{(s-2)(n+1)}\left\{\sin \theta\left(\widetilde{\sigma}_{r}\left(\left(\tilde{u}_{\theta}\right)-\left(\tilde{u}_{r}\right)^{*}\right)-\widetilde{\sigma}_{r \theta}\left(\left(\tilde{u}_{\theta}\right)^{\bullet}+\tilde{u}_{r}\right)\right)\right. \\
\left.+(n(s-2)+1) \cos \theta\left(\widetilde{\sigma}_{r} \tilde{u}_{r}+\tilde{\sigma}_{r \theta} \tilde{u}_{\theta}\right)\right\}, \\
i=x, y, z, j=x, y .
\end{gathered}
$$

Here, taking strain-displacement relations into account, $\tilde{u}_{r},\left(\tilde{u}_{r}\right)^{\bullet},\left(\tilde{u}_{\theta}\right)^{\bullet}$ and $\tilde{u}_{\theta}$ may be expressed as

$$
\begin{aligned}
& u_{r}=\int \varepsilon_{r} d r=\frac{\alpha}{2} \int K^{n} r^{(s-2) n} \widetilde{\varepsilon}_{r} d r=\frac{\alpha K^{n_{r}(s-2) n+1} \widetilde{\varepsilon}_{r}}{2(n(s-2)+1)}=\alpha K^{n} r^{(s-2) n+1} \widetilde{u}_{r}(\theta, z) \\
& \left(u_{r}\right)^{*}=\frac{\alpha K^{n_{r}(s-2) n+1}}{2((s-2) n+1)}\left(\widetilde{\varepsilon}_{r}\right)^{*}=\alpha K^{n} r^{(s-2) n+1}\left(\widetilde{u}_{r}(\theta, z)\right)^{*}, \\
& \left(u_{\theta}\right)^{\bullet}=\varepsilon_{\theta} r-u_{r}=\alpha K^{n} r^{(s-2) n+1}\left(\widetilde{\varepsilon}_{\theta}-\tilde{u}_{r}\right)=\alpha K^{n} r^{(s-2) n+1}\left(\tilde{u}_{\theta}(\theta, z)\right)^{\bullet}, \\
& u_{\theta}=-\alpha K^{n} r^{(s-2) n+1} \frac{\left(3 \tilde{\sigma}_{e}^{n-1}(s-1) \widetilde{\phi}^{*}+\left(\widetilde{u}_{r}\right)^{*}\right)}{n(s-2)}=\alpha K^{n} r^{(s-2) n+1} \widetilde{u}_{\theta}(\theta, z) .
\end{aligned}
$$

Here, the last term in Eq. (26) may be obtained by the following expression, written as

$$
\frac{1}{2}\left(\frac{1}{r}\left(u_{r}\right)^{\cdot}+\left(u_{\theta}\right)^{\prime}-\frac{u_{\theta}}{r}\right)=\varepsilon_{r \theta}
$$

Substituting $\left(u_{r}\right)^{\bullet}=\alpha K^{n} r^{(s-2) n+1}\left(\tilde{u}_{r}\right)^{\bullet}, u_{\theta}=\alpha K^{n} r^{(s-2) n+1} \tilde{u}_{\theta}$ and $\varepsilon_{r \theta}=-\frac{3 \alpha}{2} K^{n} r^{(s-2) n} \widetilde{\sigma}_{e}^{n-1}(s-1) \tilde{\phi}^{\bullet}$ into Eq. (27), one may have

$$
\tilde{u}_{\theta}=-\frac{3 \widetilde{\sigma}_{e}^{n-1}(s-1) \tilde{\phi}^{\cdot}+\left(\tilde{u}_{r}\right)^{\cdot}}{(s-2) n}
$$

Substituting Eq. (26) into Eq. (25), then substituting Eq. (24) and Eq. (25) into Eq. (23), the $J$-integral on $\Gamma$ may be written as

$$
\begin{gathered}
J=\int_{\Gamma}\left(W d y-\sigma_{i j} n_{j} u_{i, x} d s\right)=\alpha K^{n+1} r^{(s-2)(n+1)+1} I,(r \rightarrow 0), \\
I=\int_{-\pi}^{\pi}\left\{\frac{n}{n+1}\left(\widetilde{\sigma}_{e}\right)^{n+1} \cos \theta\right. \\
\left.-\left(\sin \theta\left(\widetilde{\sigma}_{r}\left(\widetilde{u}_{\theta}-\left(\widetilde{u}_{r}\right)^{*}\right)-\widetilde{\sigma}_{r \theta}\left(\left(\widetilde{u}_{\theta}\right)^{\cdot}+\tilde{u}_{r}\right)\right)+\cos \theta((s-2) n+1)\left(\widetilde{\sigma}_{r} \widetilde{u}_{r}+\widetilde{\sigma}_{r \theta} \tilde{u}_{\theta}\right)\right)\right\} d \theta .
\end{gathered}
$$

When $r$ tends to zero, the three-dimensional $J$-integral should not be zero or infinity. Hence, we have

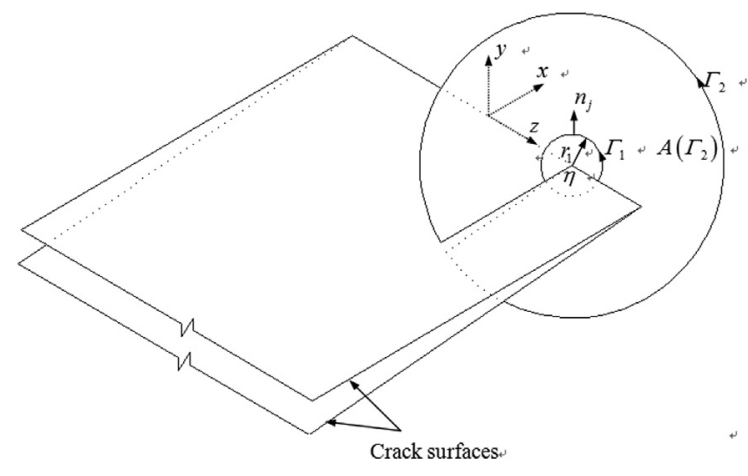

Fig. 2. Three-dimensional $J$-integral. 


$$
\left.\begin{array}{c}
J=\alpha K^{n+1} I, \\
S=\frac{2 n+1}{n+1} .
\end{array}\right\}
$$

\subsection{Solutions for $\widetilde{\sigma}_{r}, \widetilde{\sigma}_{\theta}$ and $\widetilde{\sigma}_{r \theta}$}

Considering the expressions for the strain components shown in Eq. (16), one may obtain the following expressions, written as

$$
\begin{aligned}
& \left(r \varepsilon_{\theta}\right)^{\prime \prime}=\frac{\alpha}{2}\left[\left(\sigma_{e}^{n-1}\right)^{\prime \prime}\left(2 r \phi^{\prime}-\phi^{\prime}-\frac{\phi^{*}}{r}-T\left(r \phi^{\prime \prime}+\phi^{\prime}+\frac{\phi^{\bullet \cdot}}{r}\right)\right)\right. \\
& +2\left(\sigma_{e}^{n-1}\right)^{\prime}\left(2 r \phi^{\prime \prime \prime}+\phi^{\prime \prime}-\frac{\phi^{\cdot{ }^{\prime}}}{r}+\frac{\phi^{\cdot}}{r^{2}}-T\left(r \phi^{\prime \prime}+3 \phi^{\prime \prime}+\frac{\phi^{\cdot{ }^{\prime}}}{r}-\frac{\phi^{\bullet}}{r^{2}}\right)\right)
\end{aligned}
$$

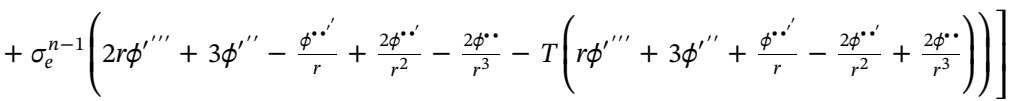

$$
\begin{aligned}
& \left(\varepsilon_{r}\right)^{\prime}=\frac{\alpha}{2}\left[\left(\sigma_{e}^{n-1}\right)^{\prime}\left(\frac{2 \phi^{\prime}}{r}+\frac{2 \phi^{\bullet}}{r^{2}}-\phi^{\prime \prime}-T\left(\frac{\phi^{\prime}}{r}+\frac{\phi^{\bullet}}{r^{2}}+\phi^{\prime}\right)\right)\right. \\
& \left.+\sigma_{e}^{n-1}\left(\frac{2 \phi^{\prime}}{r}-\frac{2 \phi^{\prime}}{r^{2}}+\frac{2 \phi^{\bullet^{\prime}}}{r^{2}}-\frac{4 \phi^{\bullet}}{r^{3}}-\phi^{\prime \prime \prime}-T\left(\frac{\phi^{\prime}}{r}-\frac{\phi^{\prime}}{r^{2}}+\frac{\phi^{\bullet^{\prime}}}{r^{2}}-\frac{2 \phi^{\bullet}}{r^{3}}+\phi^{\prime \prime \prime}\right)\right)\right] \\
& \left(\varepsilon_{r}\right)^{\cdot}=\frac{\alpha}{2}\left[\left(\sigma_{e}^{n-1}\right)^{\cdot}\left(\frac{2}{r} \phi^{\prime}+\frac{2}{r^{2}} \phi^{\bullet}-\phi^{\prime \prime}-T\left(\frac{\phi^{\prime}}{r}+\frac{\phi^{\bullet}}{r^{2}}+\phi^{\prime}\right)\right)\right. \\
& +2\left(\sigma_{e}^{n-1}\right)^{\cdot}\left(\frac{2}{r} \phi^{\dot{\prime}^{\prime}}+\frac{2}{r^{2}} \phi^{\cdots}-\phi^{\bullet^{\prime \prime}}-T\left(\frac{\phi^{\bullet^{\prime}}}{r}+\frac{\phi^{\cdots}}{r^{2}}+\phi^{\bullet^{\prime \prime}}\right)\right) \\
& \left.+\sigma_{e}^{n-1}\left(\frac{2}{r} \phi^{\bullet^{\prime}}+\frac{2}{r^{2}} \phi^{\cdots \cdots}-\phi^{\cdot{ }^{\prime \prime}}-T\left(\frac{\phi^{\cdot \cdot}}{r}+\frac{\phi^{\cdots \cdot}}{r^{2}}+\phi^{\cdot{ }^{\prime \prime}}\right)\right)\right] \\
& \left(\left(\gamma_{r \theta}\right)^{*} r\right)^{\prime}=-3 \alpha\left[\left(\sigma_{e}^{n-1}\right)^{0^{\prime}}\left(\phi^{\dot{\prime}^{\prime}}-\frac{\phi^{*}}{r}\right)+\left(\sigma_{e}^{n-1}\right) \cdot\left(\phi^{{\phi^{\prime}}^{\prime}}-\frac{\phi^{\phi^{\prime}}}{r}+\frac{\phi^{*}}{r^{2}}\right)\right. \\
& \left.+\left(\sigma_{e}^{n-1}\right)^{\prime}\left(\phi^{\cdot \bullet^{\prime}}-\frac{\phi^{\cdot \bullet}}{r}\right)+\sigma_{e}^{n-1}\left(\phi^{\cdot \cdot^{\prime}}-\frac{\phi^{\cdot{ }^{\prime}}}{r}+\frac{\phi^{*}}{r^{2}}\right)\right] \\
& \frac{1}{r}\left(\varepsilon_{z}\right)^{\cdot \cdot}+\left(\varepsilon_{z} r\right)^{\prime \prime}-\left(\varepsilon_{z}\right)^{\prime} \\
& =\alpha \frac{2 T-1}{2}\left[\left(\sigma_{e}^{n-1}\right)^{\prime \prime}\left(\left(\phi^{\prime}+\frac{1}{r} \phi^{\bullet}+r \phi^{\prime}\right)\right)+\left(\sigma_{e}^{n-1}\right)^{\prime}\left(2 r \phi^{\prime \prime \prime \prime}+3 \phi^{\prime \prime}+\frac{2 \phi^{\cdot{ }^{\prime}}}{r}-\frac{\phi^{\prime}}{r}-\frac{3 \phi^{\bullet}}{r^{2}}\right)\right. \\
& +\left(\sigma_{e}^{n-1}\right) \cdot \cdot\left(\frac{\phi^{\prime}}{r^{2}}+\frac{\phi^{\bullet}}{r^{3}}+\frac{\phi^{\prime}}{r}\right)+\left(\sigma_{e}^{n-1}\right)^{\cdot} \cdot\left(\frac{2}{r} \phi^{\prime \prime} \cdot \frac{2 \phi^{\bullet}}{r^{2}}+\frac{2 \phi^{\bullet}}{r^{3}}\right) \\
& \left.+\left(\sigma_{e}^{n-1}\right)\left(r \phi^{\prime \prime \prime \prime}+2 \phi^{\prime \prime \prime}+\frac{2 \phi^{\prime \prime} \cdot}{r}-\frac{\phi^{\prime}}{r}-\frac{2 \phi^{\prime} \cdot}{r^{2}}+\frac{\phi^{\prime}}{r^{2}}+\frac{4 \phi^{\bullet}}{r^{3}}+\frac{1}{r^{3}} \phi^{\cdots \cdot}\right)\right]
\end{aligned}
$$

Substituting Eqs. (31)-(35) into Eq. (22), one may obtain the following equations

$$
A\left(\sigma_{e}^{n-1}\right)^{\prime \prime}+B\left(\sigma_{e}^{n-1}\right)^{\cdot}+C\left(\sigma_{e}^{n-1}\right)^{\prime^{\prime}}+D\left(\sigma_{e}^{n-1}\right)^{\prime}+E\left(\sigma_{e}^{n-1}\right)^{\bullet}+F \sigma_{e}^{n-1}=0
$$

Here,

Table 1

Values of $I$ with various $T$ and $n$.

\begin{tabular}{llllllllllll}
\hline$I$ & $T$ & & & & & & & & & \\
\hline$n$ & 0 & 0.05 & 0.1 & 0.15 & 0.2 & 0.25 & 0.3 & 0.35 & 0.4 & 0.45 & 0.5 \\
\hline 3 & 3.86 & 4.14 & 4.46 & 4.80 & 5.12 & 5.38 & 5.46 & 5.39 & 5.36 & 5.43 \\
5 & 3.41 & 3.71 & 4.03 & 4.40 & 4.76 & 5.08 & 4.89 & 4.73 & 4.81 & 4.91 \\
9 & 3.04 & 3.31 & 3.66 & 4.07 & 4.45 & 4.85 & 4.27 & 4.21 & 4.37 & 4.49 \\
13 & 2.87 & 3.14 & 3.51 & 3.96 & 4.40 & 4.70 & 4.00 & 4.01 & 4.14 & 4.29 \\
\hline
\end{tabular}




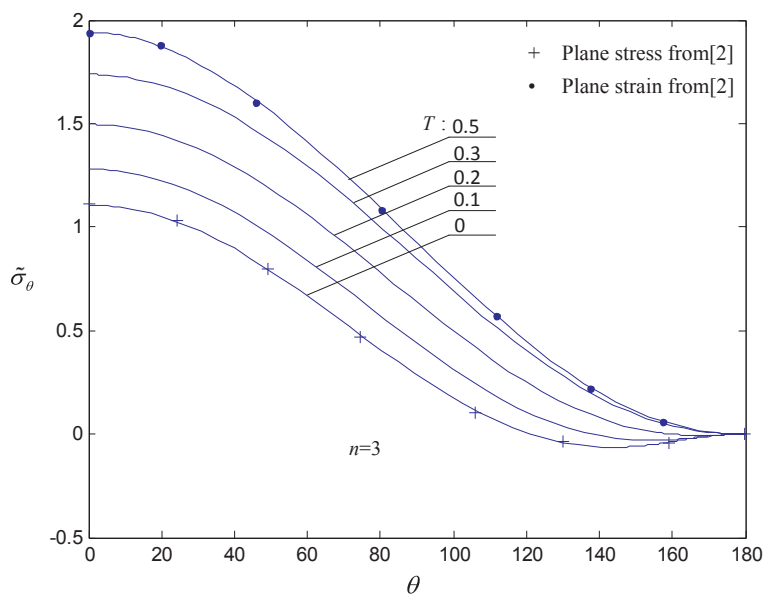

(a)

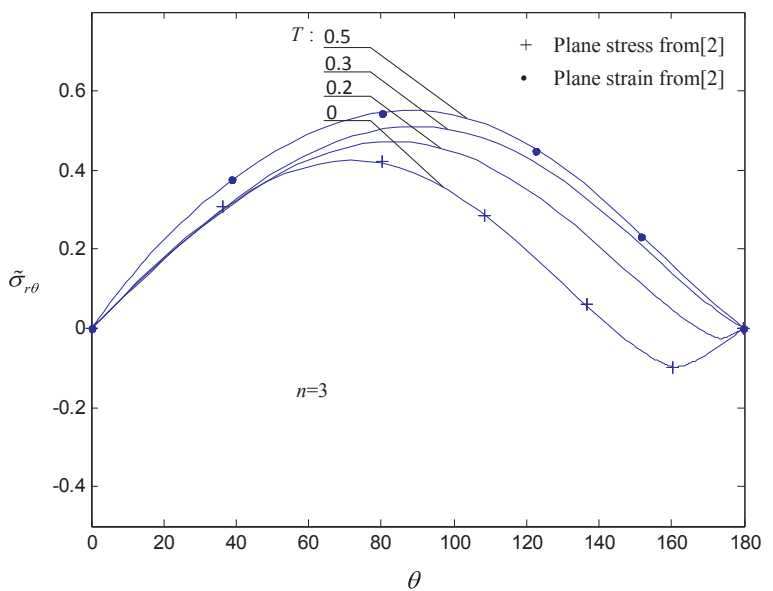

(c)

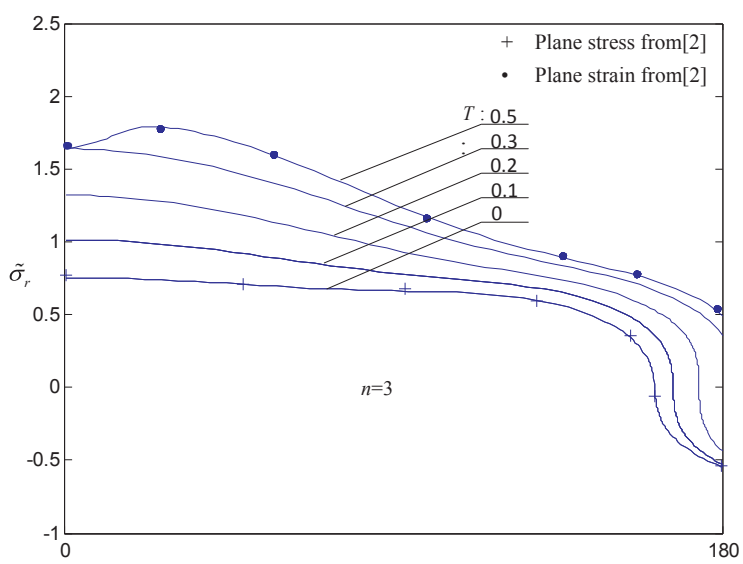

(b)

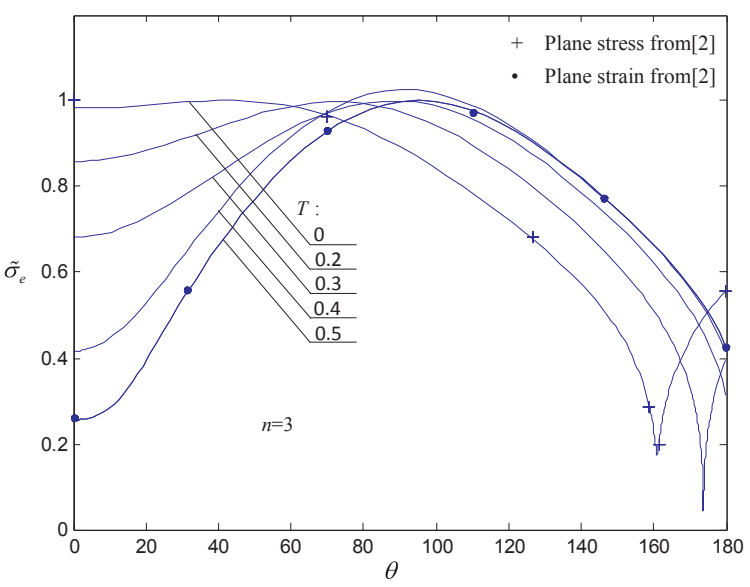

(d)

Fig. 3. Effects of the out-of-plane constraint level $T$ on the in-plane stresses when $n$ is 3. (a) $\theta$-variation of $\widetilde{\sigma}_{\theta}$, (b) $\theta$-variation of $\widetilde{\sigma}_{r}$, (c) $\theta$-variation of $\widetilde{\sigma}_{r \theta}$ and (d) $\theta$-variation of $\widetilde{\sigma}_{e}$.

$$
\begin{aligned}
& A=2 r \phi^{\prime \prime}-\phi^{\prime}-\frac{\phi^{*}}{r}+2 T(T-1)\left(r \phi^{\prime \prime}+\phi^{\prime}+\frac{\phi^{*}}{r}\right), \\
& B=-\frac{\phi^{\prime \prime}}{r}+\frac{2 \phi^{\prime}}{r^{2}}+\frac{2 \phi^{*}}{r^{3}}+2 T(T-1)\left(\frac{\phi^{\prime \prime}}{r}+\frac{\phi^{\prime}}{r^{2}}+\frac{\phi^{*}}{r^{3}}\right) \text {, } \\
& C=6\left(\frac{\phi^{\prime}}{r}-\frac{\phi^{*}}{r^{2}}\right) \text {, } \\
& D=4 r \phi^{\prime \prime \prime}+3 \phi^{\prime \prime}+\frac{4 \phi^{*^{\prime}}}{r}-\frac{2 \phi^{\prime}}{r}-\frac{6 \phi^{\circ}}{r^{2}}+2 T(T-1)\left(2 r \phi^{\prime \prime \prime}+3 \phi^{\prime \prime}+\frac{2 \phi^{\circ{ }^{\prime}}}{r}-\frac{\phi^{\prime}}{r}-\frac{3 \phi^{\circ}}{r^{2}}\right), \\
& E=\frac{4 \phi^{\prime}}{r}-\frac{2 \phi^{\prime}}{r^{2}}+\frac{4 \phi^{\cdots}}{r^{3}}+\frac{6 \phi^{\circ}}{r^{3}}+4 T(T-1)\left(\frac{\phi^{\phi^{\prime}}}{r}+\frac{\phi^{\prime}}{r^{2}}+\frac{\phi^{\cdots} \cdot}{r^{3}}\right), \\
& F=(2+2 T(T-1))\left(r \phi^{\prime \prime \prime \prime}+2 \phi^{\prime \prime \prime}+\frac{2 \phi^{\bullet^{\prime}}}{r}-\frac{\phi^{\prime \prime}}{r}-\frac{2 \phi^{\bullet^{\prime}}}{r^{2}}+\frac{\phi^{\prime}}{r^{2}}+\frac{4 \phi^{\bullet}}{r^{3}}+\frac{\phi^{\cdots \cdots}}{r^{3}}\right) .
\end{aligned}
$$

Considering Eq. (17), one may obtain the following expressions, written as 


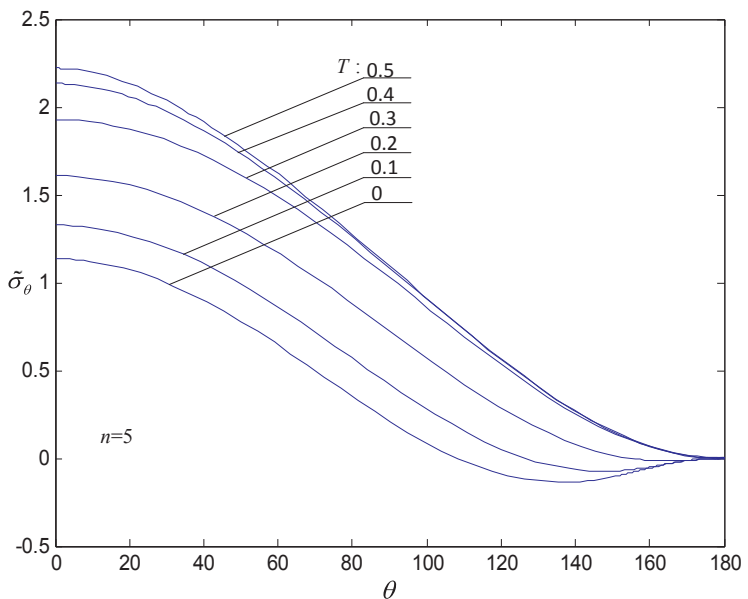

(a)

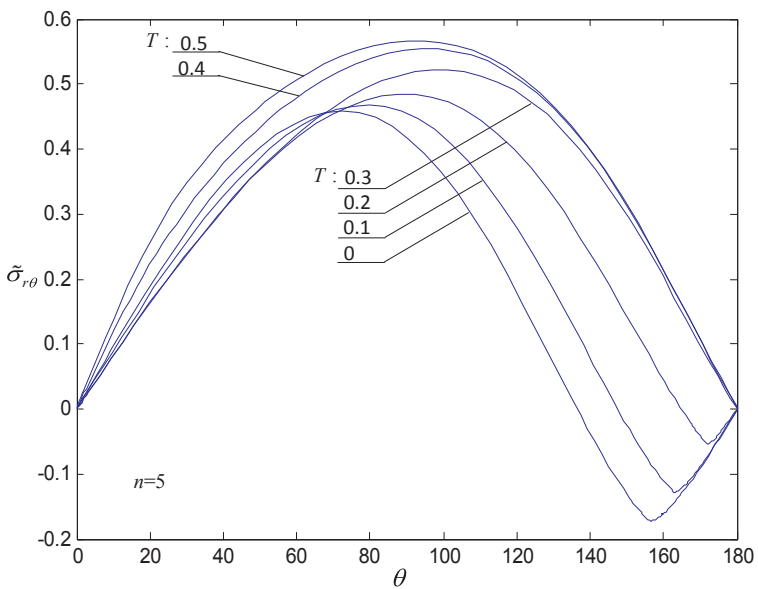

(c)

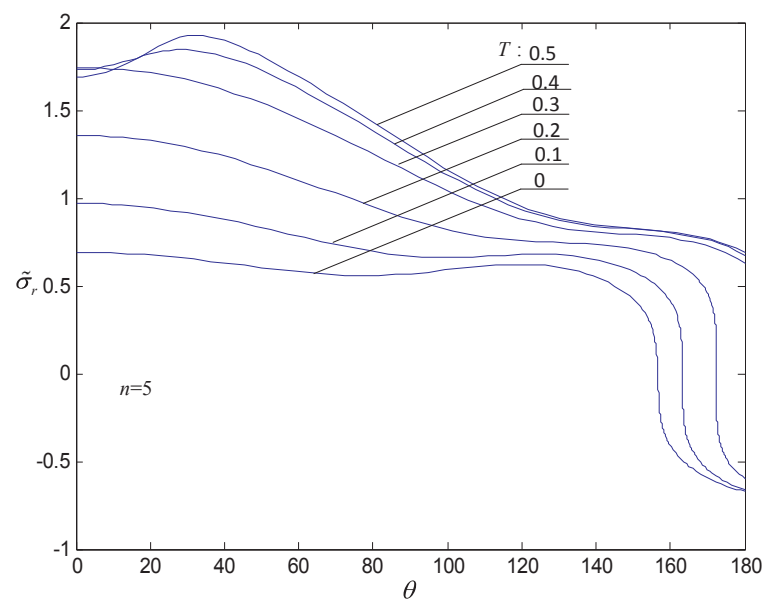

(b)

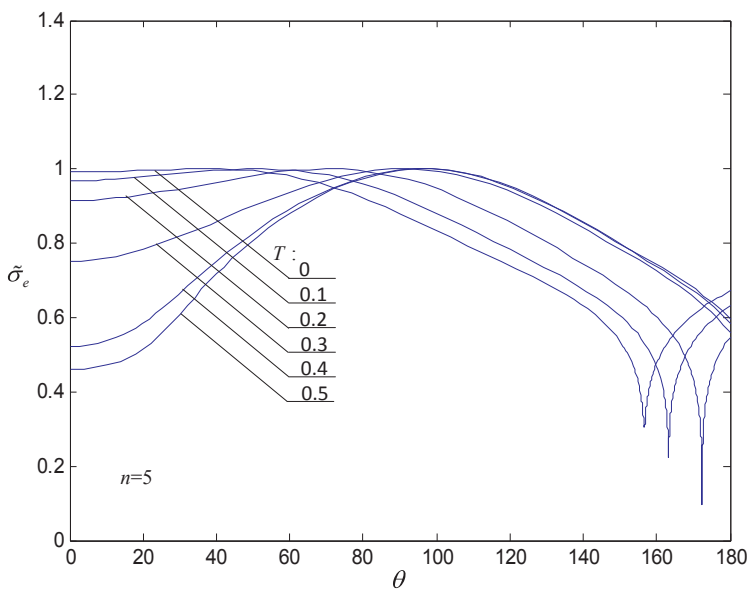

(d)

Fig. 4. Effects of the out-of-plane constraint level $T$ on the in-plane stresses when $n$ is 5. (a) $\theta$-variation of $\widetilde{\sigma}_{\theta}$, (b) $\theta$-variation of $\widetilde{\sigma}_{r}$, (c) $\theta$-variation of $\widetilde{\sigma}_{r \theta}$ and (d) $\theta$-variation of $\tilde{\sigma}_{e}$.

$$
\left.\begin{array}{c}
\left(\sigma_{e}^{n-1}\right)^{\prime}=\left(K^{n-1} r^{(s-2)(n-1)} \widetilde{\sigma}_{e}^{n-1}\right)^{\prime}=K^{n-1}(s-2)(n-1) r^{(s-2)(n-1)-1} \widetilde{\sigma}_{e}^{n-1}, \\
\left(\sigma_{e}^{n-1}\right)^{\prime \prime}=K^{n-1}(s-2)(n-1)((s-2)(n-1)-1) r^{(s-2)(n-1)-2} \widetilde{\sigma}_{e}^{n-1}, \\
\left(\sigma_{e}^{n-1}\right)^{\cdot}=\left(K^{n-1} r^{(s-2)(n-1)} \widetilde{\sigma}_{e}^{n-1}\right)^{\bullet}=K^{n-1} r^{(s-2)(n-1)}(n-1) \widetilde{\sigma}_{e}^{n-2}\left(\widetilde{\sigma}_{e}\right)^{\bullet}, \\
\left(\sigma_{e}^{n-1}\right)^{\prime}=K^{n-1}(s-2)(n-1)^{2} r^{(s-2)(n-1)-1} \widetilde{\sigma}_{e}^{n-2}\left(\widetilde{\sigma}_{e}\right)^{\cdot}, \\
\left(\sigma_{e}^{n-1}\right)^{\cdot *}=K^{n-1} r^{(s-2)(n-1)}(n-1)\left(\widetilde{\sigma}_{e}^{n-2}\left(\widetilde{\sigma}_{e}\right)^{*}\right)^{*} \\
=K^{n-1} r^{(s-2)(n-1)}(n-1)\left((n-2) \widetilde{\sigma}_{e}^{n-3}\left(\left(\widetilde{\sigma}_{e}\right)^{*}\right)^{2}+\widetilde{\sigma}_{e}^{n-2}\left(\widetilde{\sigma}_{e}\right)^{*}\right) .
\end{array}\right\}
$$

Here,

$$
\left.\begin{array}{c}
\left(\widetilde{\sigma}_{e}\right)^{\cdot}=\frac{1}{2 \widetilde{\sigma}_{e}}\left(\widetilde{\sigma}_{e}^{2}\right)^{\cdot}, \\
\left.\left(\widetilde{\sigma}_{e}\right)^{*}=\frac{1}{2 \widetilde{\sigma}_{e}}\left(\left(\widetilde{\sigma}_{e}^{2}\right)^{\cdot \cdot}-\frac{1}{2 \widetilde{\sigma}_{e}^{2}}\left(\left(\widetilde{\sigma}_{e}^{2}\right)^{\cdot}\right)^{2}\right) \cdot\right\}
\end{array}\right\}
$$

Substituting Eq. (38), Eq. (39) into Eq. (36) and Substituting $\phi=K r^{s} \tilde{\phi}$ into Eq. (37), one may obtain the following equation, written as .

$$
\begin{gathered}
\widetilde{\phi}^{\cdots \cdots}\left(\widetilde{\phi}, \widetilde{\phi}^{\cdot}, \widetilde{\phi}^{\cdots}, \widetilde{\phi}^{\cdots}, T, n\right)=\frac{1}{H}\left[\widetilde{B}(n-1)\left((n-3) \frac{1}{4 \tilde{\sigma}_{e}^{2}}\left(\left(\widetilde{\sigma}_{e}^{2}\right)^{\cdot}\right)^{2}+\frac{1}{2}\left(\widetilde{\sigma}_{e}^{2}\right)^{\cdot *}\right)\right. \\
+\frac{(n-1)}{2}(\widetilde{C}(s-2)(n-1)+\widetilde{E})\left(\widetilde{\sigma}_{e}^{2}\right)^{\cdot} \\
\left.+(\widetilde{A}(s-2)(n-1)((s-2)(n-1)-1)+\widetilde{D}(s-2)(n-1)+\widetilde{F}) \widetilde{\sigma}_{e}^{2}\right] .
\end{gathered}
$$




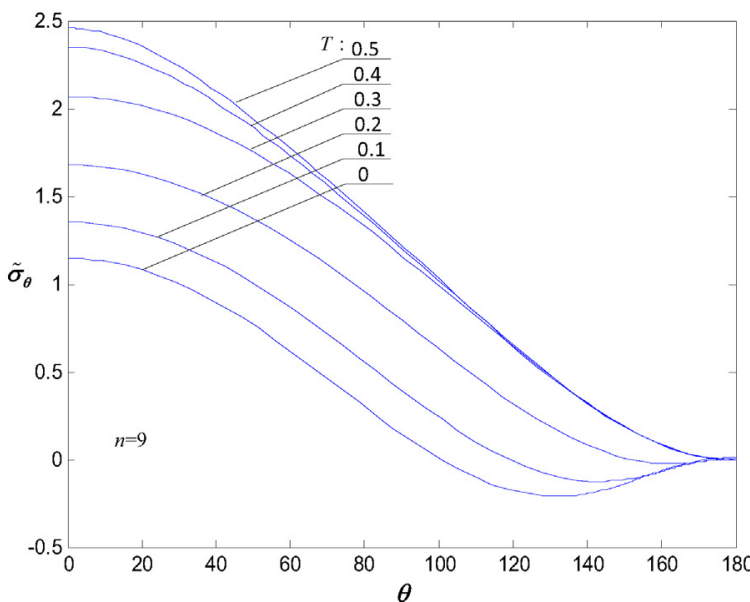

(a)

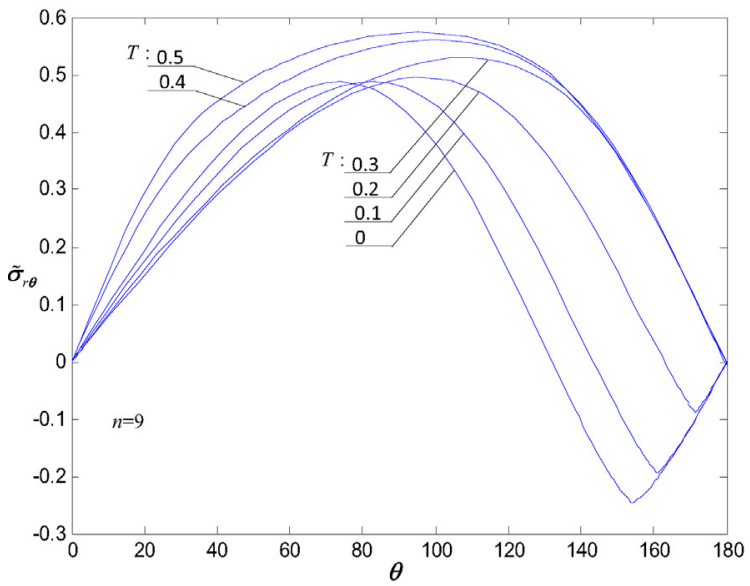

(c)

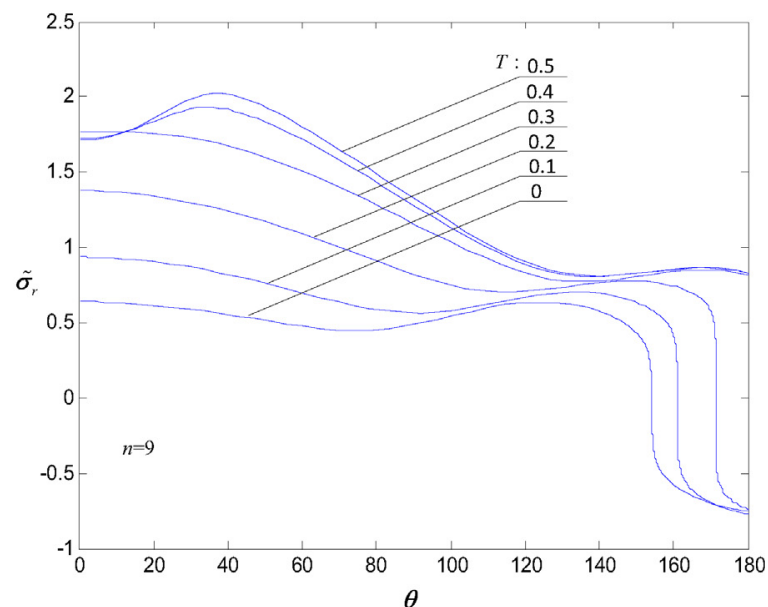

(b)

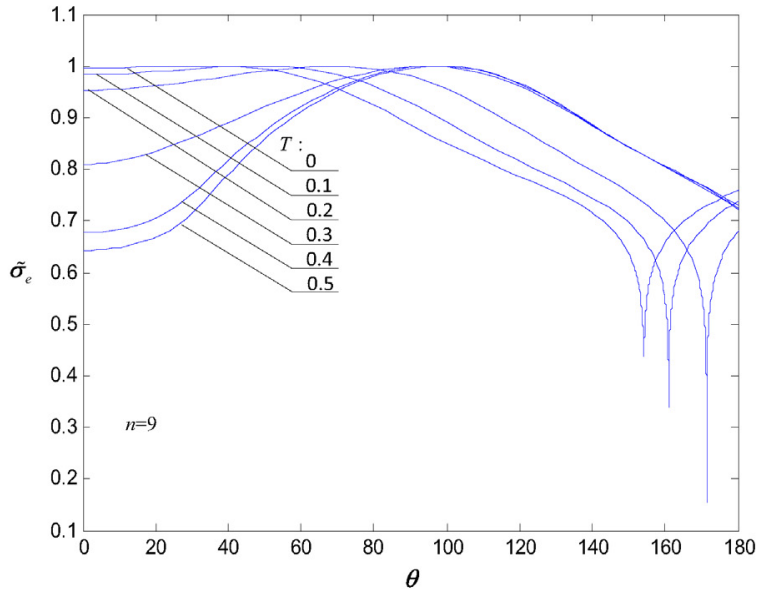

(d)

Fig. 5. Effects of the out-of-plane constraint level $T$ on the in-plane stresses when $n$ is 9. (a) $\theta$-variation of $\widetilde{\sigma}_{\theta}$, (b) $\theta$-variation of $\widetilde{\sigma}_{r}$, (c) $\theta$-variation of $\widetilde{\sigma}_{r \theta}$ and (d) $\theta$-variation of $\widetilde{\sigma}_{e}$.

Here,

$$
\begin{aligned}
& H=-\frac{3 \widetilde{B}(n-1)}{2}\left(\left(1-\frac{1+T}{3}(s-1)\right) s \tilde{\phi}+\widetilde{\phi} \cdot \cdot+\frac{T(2 T-1)}{3}\left(\widetilde{\phi} \cdot \cdot+s^{2} \tilde{\phi}\right)\right) \\
& -2(1+(T-1) T) \tilde{\sigma}_{e}^{2}, \\
& \widetilde{A}=\left(2 s^{2}-3 s\right) \widetilde{\phi}-\widetilde{\phi}^{\cdot \cdot}+2 T(T-1)\left(\tilde{\phi}^{\cdot}+s^{2} \widetilde{\phi}\right), \\
& \widetilde{B}=2 \widetilde{\phi}^{\cdot \cdot}+s(3-s) \tilde{\phi}+2 T(T-1)\left(\tilde{\phi}^{\cdot}+s^{2} \widetilde{\phi}\right) \text {, } \\
& \widetilde{C}=6(s-1) \tilde{\phi}, \\
& \widetilde{D}=s\left(4 s^{2}-9 s+3\right) \tilde{\phi}+2(2 s-3) \tilde{\phi}^{*}+2 T(T-1)\left(s\left(2 s^{2}-3 s\right) \widetilde{\phi}+(2 s-3) \widetilde{\phi}^{*}\right) \text {, } \\
& \widetilde{E}=2\left(2 s^{2}-3 s+3\right) \tilde{\phi}^{\cdot}+4 \tilde{\phi}^{\cdots}+2 T(T-1)\left(2 \widetilde{\phi}^{\cdots}+2 s^{2} \widetilde{\phi}^{*}\right) \text {, } \\
& \widetilde{F}=2\left(T^{2}-T+1\right)\left(s^{2}(s-2)^{2} \widetilde{\phi}+2\left(s^{2}-2 s+2\right) \widetilde{\phi}^{*}\right) \text {. } \\
& \left(\widetilde{\sigma}_{e}^{2}\right)^{\cdot}=3\left[\left(s(s-1) \widetilde{\phi}-\frac{1+T}{3}\left(\widetilde{\phi}^{*}+s^{2} \widetilde{\phi}\right)\right)\left(s(s-1) \tilde{\phi}^{\cdot}-\frac{1+T}{3}\left(\tilde{\phi}^{\cdots}+s^{2} \widetilde{\phi}^{*}\right)\right)\right. \\
& +\left(s \tilde{\phi}+\widetilde{\phi}^{\cdot}-\frac{1+T}{3}\left(\tilde{\phi}^{\cdot}+s^{2} \widetilde{\phi}\right)\right)\left(s \tilde{\phi}^{\cdot}+\widetilde{\phi}^{\cdots}-\frac{1+T}{3}\left(\widetilde{\phi}^{\cdots}+s^{2} \widetilde{\phi}^{*}\right)\right) \\
& +\left(\frac{2 T-1}{3}\right)^{2}\left(\widetilde{\phi}^{\cdot}+s^{2} \widetilde{\phi}\right)\left(\tilde{\phi}^{\cdots}+s^{2} \widetilde{\phi}^{*}\right)+2\left(1-s^{2} \tilde{\phi}^{\cdot} \tilde{\phi}^{\cdot}\right]
\end{aligned}
$$

and 


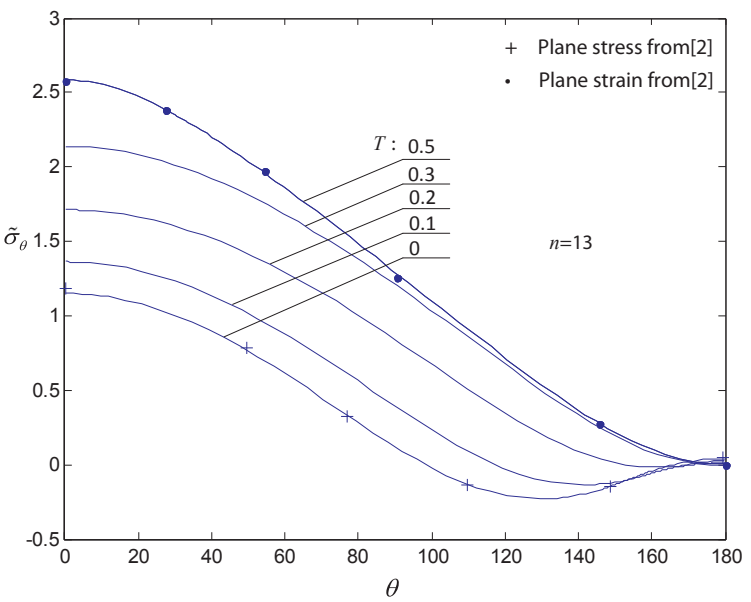

(a)

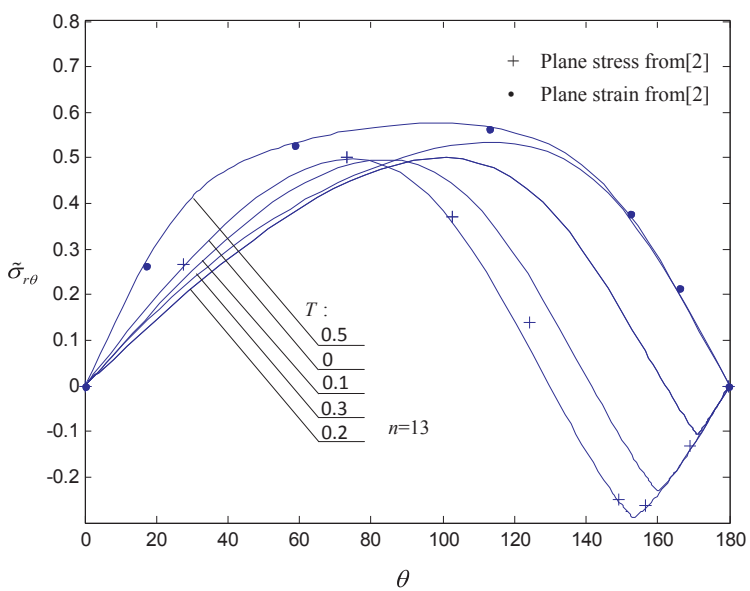

(c)

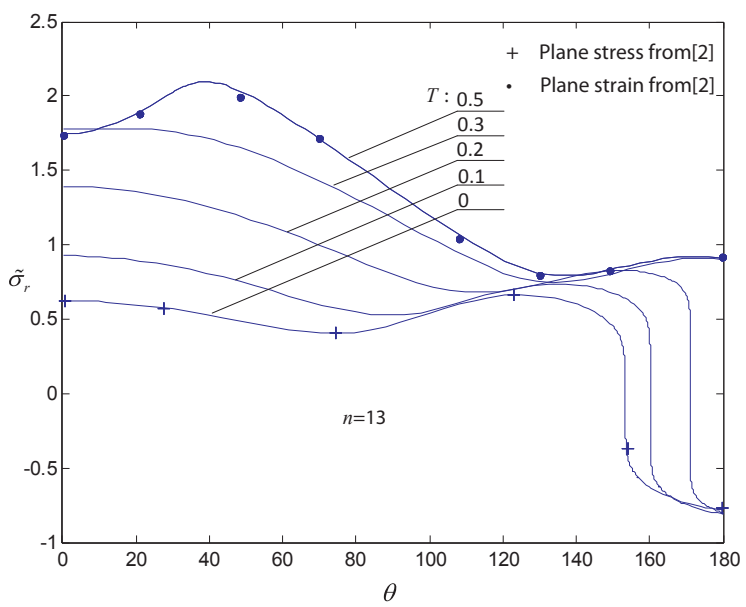

(b)

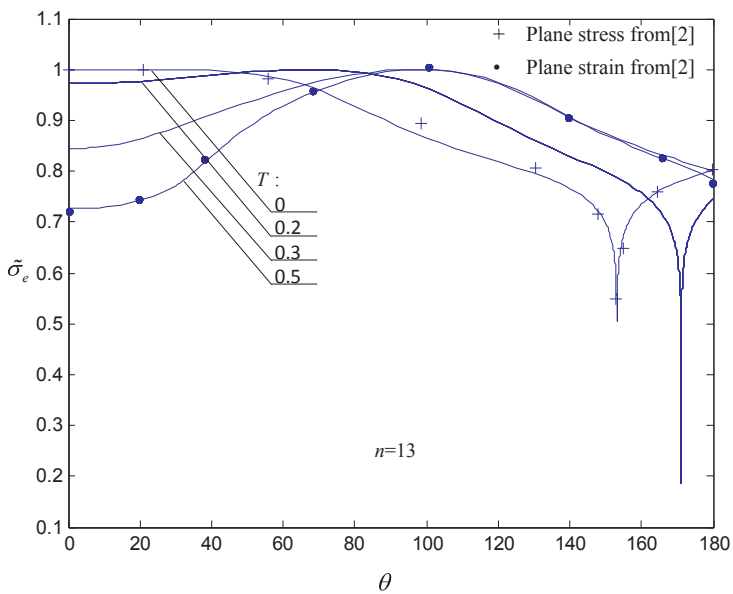

(d)

Fig. 6. Effects of the out-of-plane constraint level $T$ on the in-plane stresses when $n$ is 13. (a) $\theta$-variation of $\widetilde{\sigma}_{\theta}$, (b) $\theta$-variation of $\widetilde{\sigma}_{r}$, (c) $\theta$-variation of $\widetilde{\sigma}_{r \theta}$ and (d) $\theta$-variation of $\widetilde{\sigma}_{e}$.

$$
\begin{aligned}
& \left(\widetilde{\sigma}_{e}^{2}\right)^{\cdots^{*}}=3\left[\left(s(s-1) \tilde{\phi}^{\cdot}-\frac{1+T}{3}\left(\widetilde{\phi}^{\cdots}+s^{2} \widetilde{\phi}^{*}\right)\right)^{2}\right. \\
& +\left(s(s-1) \tilde{\phi}-\frac{1+T}{3}\left(\widetilde{\phi}^{\cdot}+s^{2} \widetilde{\phi}\right)\right)\left(s(s-1)-\frac{1+T}{3} s^{2}\right) \tilde{\phi}^{\cdot} \\
& +\left(s \tilde{\phi}^{\cdot}+\tilde{\phi}^{\cdots}-\frac{1+T}{3}\left(\tilde{\phi}^{\cdots}+s^{2} \tilde{\phi}^{*}\right)\right)^{2} \\
& +\left(s \tilde{\phi}+\widetilde{\phi}^{\cdot}-\frac{1+T}{3}\left(\tilde{\phi}^{\cdot}+s^{2} \widetilde{\phi}\right)\right)\left(s-\frac{1+T}{3} s^{2}\right) \tilde{\phi}^{\cdot} \\
& +\left(\frac{2 T-1}{3}\right)^{2}\left(\widetilde{\phi}^{\cdots}+s^{2} \widetilde{\phi}^{*}\right)^{2}
\end{aligned}
$$

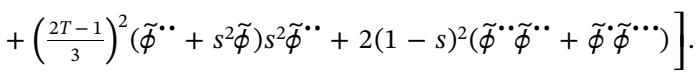

Eq. (40) can be solved by a fourth-order Runge-Kutta method when $T$ and $n$ are given. Following Hutchison's paper [2], the values of $\widetilde{\phi}(0)$ and $\widetilde{\phi}^{*}(0)$ can be determined when stress-free boundary conditions on the crack surfaces and an additional condition that $\max \left\{\tilde{\sigma}_{e}\right\}=1$ are satisfied at the same time.

Stress-free boundary conditions on the crack surfaces $\left(\sigma_{\theta}( \pm \pi)=\sigma_{r \theta}( \pm \pi)=0\right)$ require

$$
\tilde{\phi}( \pm \pi)=\widetilde{\phi}^{\cdot}( \pm \pi)=0
$$

The imposed symmetry $\left(\left(\sigma_{r \theta}\right)_{\theta=0}=\left(\sigma_{r}\right)^{\bullet}{ }_{\theta=0}=\left(\sigma_{\theta}\right)_{\theta=0}^{\cdot}=\left(\sigma_{z}\right)^{\cdot}{ }_{\theta=0}=0\right)$ requires

$$
\widetilde{\phi}^{\cdots}(0)=\tilde{\phi}^{\cdot}(0)=0
$$

After the numerical results of $\widetilde{\phi}, \widetilde{\phi}^{*}, \widetilde{\phi}^{*}$ and $\widetilde{\phi}^{\cdots}$ are obtained, one may substitute those results into Eq. (14) and Eq. (16) to obtain 


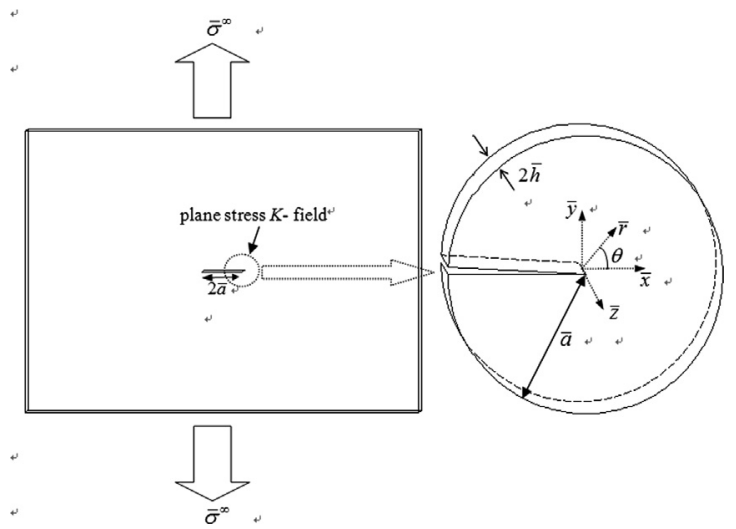

(a)

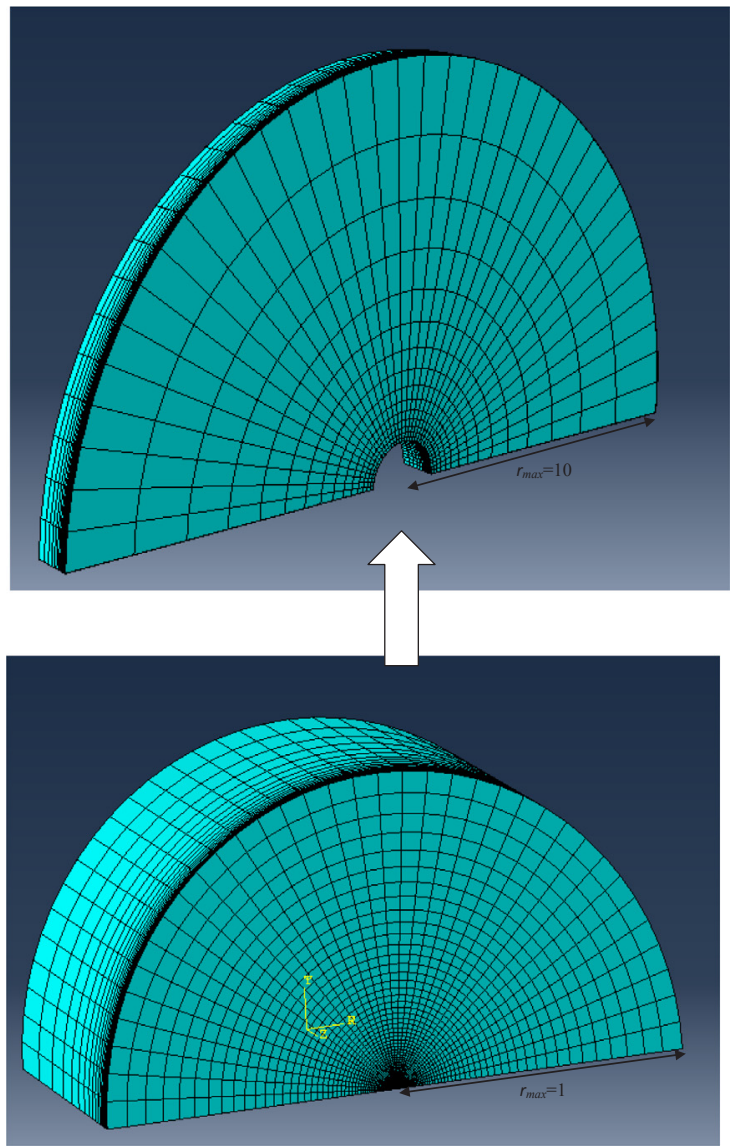

(b)

Fig. 7. Finite element simulation for a thin cracked plate with thickness $2 \bar{h}$ subjected remote tensile loading $\bar{\sigma}^{\infty}$. Here, $\bar{a}=10 \bar{h}$. (a) Schematic of a cracked circular disk which represents the near crack front region of a thin plate. (b) finite element mesh of the quarter-mode.

the corresponding numerical results of the crack front stress and stain fields. Further, one may also obtain the numerical results of $\tilde{u}_{r}$, $\left(\widetilde{u}_{r}\right)^{\bullet},\left(\tilde{u}_{\theta}\right)^{*}, \tilde{u}_{\theta}$ and $I$. The numerical results of $I, \widetilde{\sigma}_{\theta}, \widetilde{\sigma}_{r}, \widetilde{\sigma}_{r \theta}$ and $\widetilde{\sigma}_{e}$ for various $T$ and $n$ have been shown in Table.1 and Figs. 3-6.

\section{Three dimensional finite element simulations}

We consider a thin plate (the thickness is $2 \bar{h}$ ) containing a long crack(the length of the crack is $2 \bar{a}$ ) under remote tension. The near crack front region of the thin plate may be modeled by a circular disk (cylinder), as shown in Fig. 7(a). Only a quarter of circular disk (region $0 \leq \theta \leq \pi, 0 \leq \bar{z} / \bar{h} \leq 1$ ) is modeled with finite elements since the symmetry of the current problem, as shown in Fig. 7(b). The 


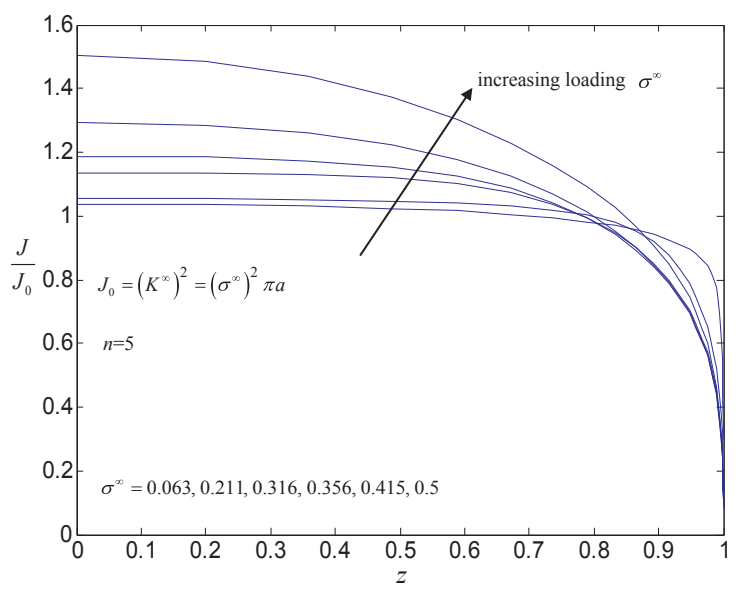

(a)

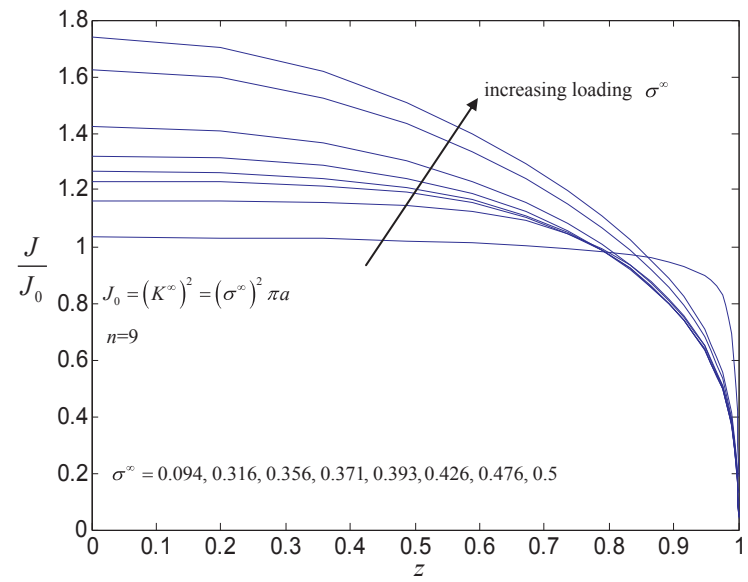

(b)

Fig. 8. Variations of $J / J_{0}$ in the thickness direction with various loading levels. Here, $a=10$. (a) $n=5$, (b) $n=9$.

finite element mesh is constructed with 8-node brick elements. It contains inner and outer regions as shown in Fig. 7(b). Within each region, in the plane perpendicular to the crack front, the element size is gradually increased with increasing radial distance from the crack front (the progression ratios are 1.07 in the inner region and 1.24 in the outer region.), while the angular increment of each element is kept constant, $\Delta \theta=\frac{\pi}{36}$. The identical planar mesh is repeated along the $\bar{z}$ axis from the symmetry plane $(\bar{z}=0)$ to the freesurface $(\bar{z}=\bar{h})$. The thickness of successive element layers gradually increases towards the symmetry plane (the progression ratio is 1.25). There are 25 layers through the half thickness of the plate, and the thickness of the layer at the free surface is $10^{-3} \bar{h}$. The radial size of the elements around the crack front is $10^{-4} \bar{h}$. The entire mesh consists of 91,800 elements.

The symmetry conditions require:

1. the normal displacements on the surface where $\bar{y}=0$ and $\bar{x} \geqslant 0$ should be zero,

2. and the normal displacements on the symmetry plane where $\bar{z}=0$ should be zero.

The stresses on the surface where $r=a(a=\bar{a} / \bar{h}=10)$ are given by

$$
\left.\begin{array}{c}
\sigma_{r}=\frac{K^{f a r}}{\sqrt{2 \pi a}} \frac{1}{4}\left(5 \cos \frac{\theta}{2}-\cos \frac{3}{2} \theta\right), \\
\sigma_{r \theta}=\frac{K^{f a r}}{\sqrt{2 \pi a}} \frac{1}{4}\left(\sin \frac{\theta}{2}+\sin \frac{3}{2} \theta\right), \\
\sigma_{r z}=0 . \\
K^{f a r}=\sigma^{\infty} \sqrt{\pi a}
\end{array}\right\}
$$

The material behavior of the plate follows isotropic $J_{2}$ deformation theory, and under uniaxial tension, the stress-strain relation follows the Ramberg-Osgood equation

$$
\varepsilon=\sigma+\alpha(\sigma)^{n}
$$

In the current finite element simulations, Poisson's ratio $v=0.3$, the material constant $\alpha=1$, the yield stain $\bar{\varepsilon}_{y}=0.002$ and the hardening exponents are taken as 5 and 9. 


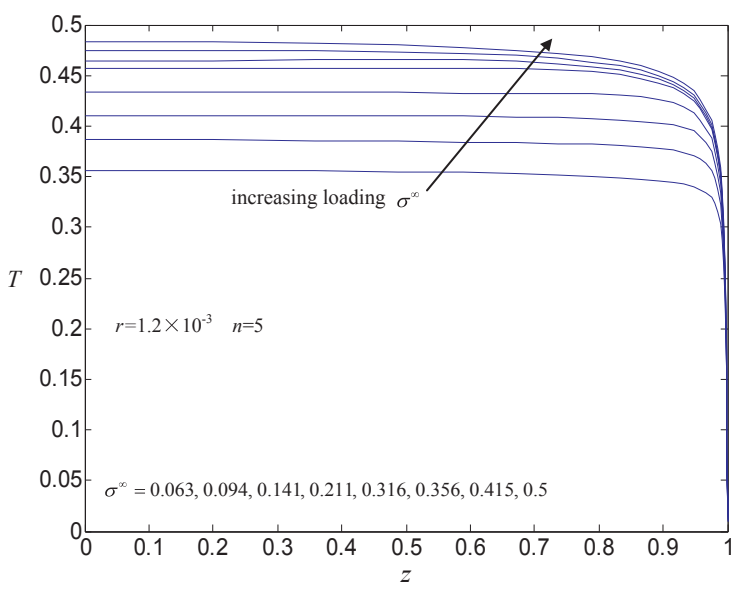

(a)

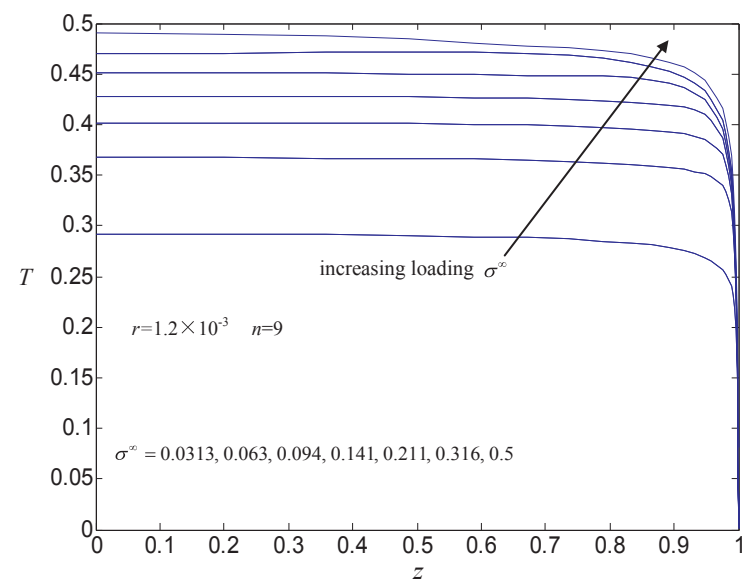

(b)

Fig. 9. Variations of the out-of-plane constraint level in the thickness direction with various loading levels. (a) $n=5$, (b) $n=9$.

\section{Results and discussions}

The effects of the out-of-plane constraint level $(T)$ on in-plane stresses $\left(\widetilde{\sigma}_{\theta}, \widetilde{\sigma}_{r}, \widetilde{\sigma}_{r \theta}\right)$ and the effective stress $\widetilde{\sigma}_{e}$ with various stain hardening coefficients are plotted in Figs. 3-6. The stain hardening coefficient $n$ is taken as 3, 5, 9 and 13 . These figures show that the value of $\tilde{\sigma}_{\theta}$ increases with increasing $T$. Fig. 3 and Fig. 6 show that when $T=0$ and $T=0.5$, the current results can reduce to the corresponding plane stress and plane strain results given by Hutchinson [2].

A fine three-dimensional finite element mesh is constructed for a thin ductile plate with a through-thickness crack under remote tension. The variations of the normalized $J$-integral $J / J_{0}, T$ and the opening stress $\sigma_{\theta}$ near the crack front in the thickness direction haven been plotted in Fig. 8, Fig. 9 and Fig. 10 separately. Fig. 8 shows that the value of $J / J_{0}$ decreases slowly first, then drops rapidly near the free surface. Fig. 8 also demonstrates that the value of $J / J_{0}$ increases with increasing remote loading $\sigma^{\infty}$ on the mid-plane. When the loading level is given, the value of $J / J_{0}$ increases with increasing stain hardening coefficient $n$.

Fig. 9 shows the variations of $T$ in the thickness direction with various remote loading $\sigma^{\infty}$. The results in Fig. 9 demonstrates that the value of $T$ decreases by less than $10 \%$ in the range of $0<z$ less than 0.95 and falls quickly to zero when approaching the free surface. Fig. 9 illustrates that the value of $T$ increases with increasing $\sigma^{\infty}$. On the mid-plane, the value of $T$ increases from 0.36 to 0.48 when the remote loading $\sigma^{\infty}$ increases from 0.065 to 0.5 . Fig. 9 also shows that the value of $T$ is higher in a soft material ( $\left.n=9\right)$ than that in a hard material $(n=5)$ when a given remote loading is applied. An explanation for the observation is that higher loading levels or higher stain hardening coefficients will cause larger plastic deformations near the crack front, which result in the decrease of the out-of-plane constraint level.

Fig. 10 shows that the value of the opening stress $\sigma_{\theta}$ decreases with increasing $z$ and lies between the corresponding plane stress and plane strain solutions given by Hutchinson [2]. This implies that the decrease of the out-of-plane constraint level $T$ will result in the decrease of $\sigma_{\theta}$. Fig. 10 also shows that the current analytical results are in good agreement with the corresponding finite element results.

The comparison of analytical and numerical results for the $\theta$-variation of in-plane stresses $\left(\sigma_{\theta}, \sigma_{r}\right.$ and $\left.\sigma_{r \theta}\right)$ near the crack front on 


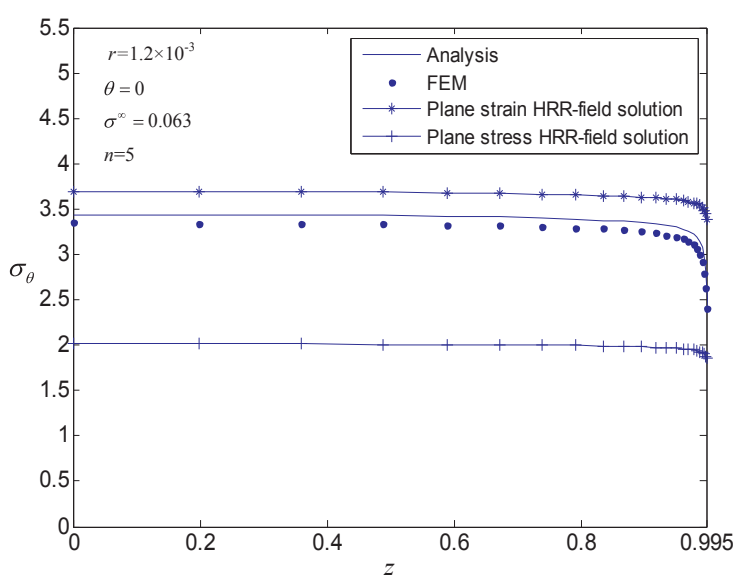

(a)

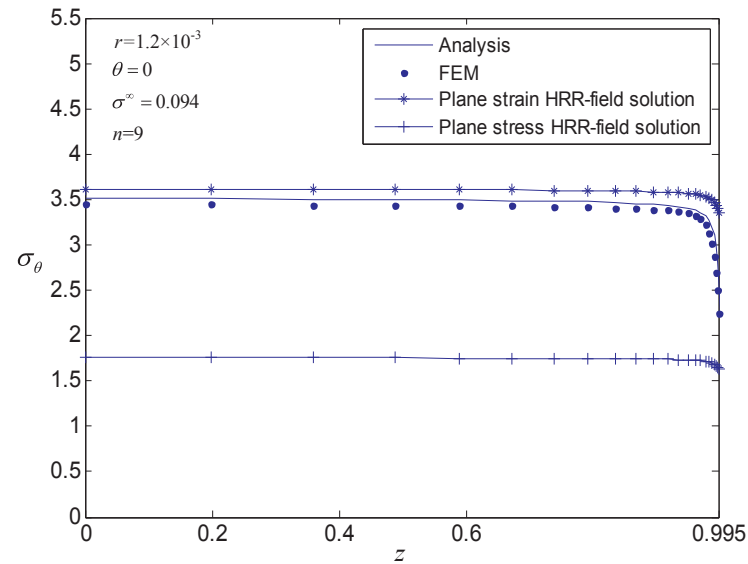

(b)

Fig. 10. Variations of the opening stress in the thickness direction. (a) $n=5$, (b) $n=9$.

the mid-plane is illustrated in Fig. 11. Analytical results are in good agreement with the corresponding finite element results

The values of $I$ for various $T$ and $n$ have been shown in Table 1 . When $T$ is taken as 0 and 0.5 separately, the values of $I$ are in good agreement with the corresponding values from plane stress and strain solutions given by Hutchinson [2]. Table1 shows that the value of $I$ increases with deceasing hardening coefficient $n$.

\section{Summaries and conclusions}

In the current paper, a total deformation theory of plasticity, a power law hardening stress-strain relation, three-dimensional Maxwell stress functions and the minimum complementary potential energy principle are used to obtain solutions for the stress fields near the front of a crack in a thin ductile plate. A fine finite element mesh consisting of 91,800 elements is constructed to compute the three-dimensional $J$-integral, the out-of-plane constraint level $T$ and the crack front stress fields. Comparison with the finite element results shows that the analytical results are valid.

The main contribution of the current paper is to propose a semi-analytical method which is employed p obtain solutions for the crack front stress fields in the region where the plane stress and strain conditions can't be met. Considering no analytical solution and few semi-analytical solutions available for three-dimensional crack front fields in ductile materials, the current research may promote the development of the three-dimensional theory of ductile fracture.

The work supports the following conclusions

1. The current $J-T$ solutions may characterize the crack front stress fields in the region where the plane stress and strain conditions can't be met.

2. The value of the out-of-plane constraint level $T$ increases with increasing remote tensile loading, and the value of opening stress increases with increasing $T$.

3. The value of the out-of-plane constraint level $T$ is higher in a soft material $(n=9)$ than that in a hard material $(n=5)$. 


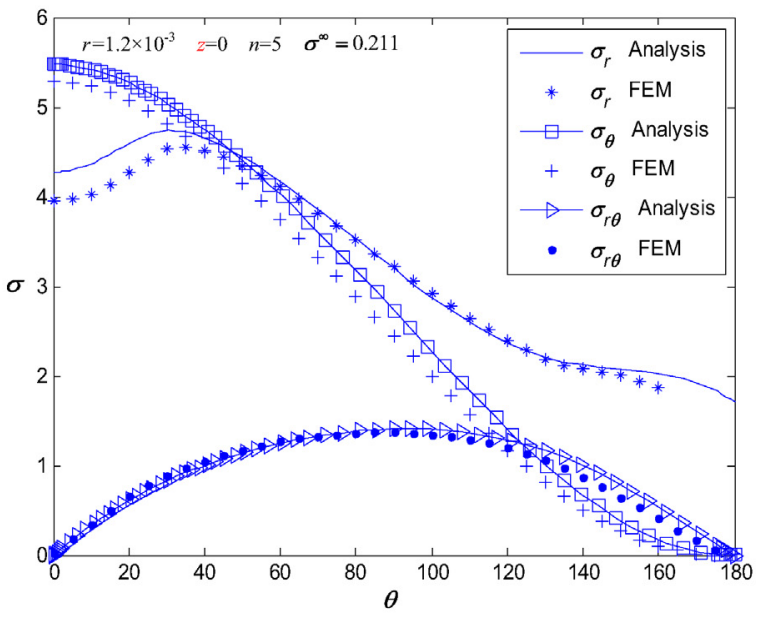

(a)

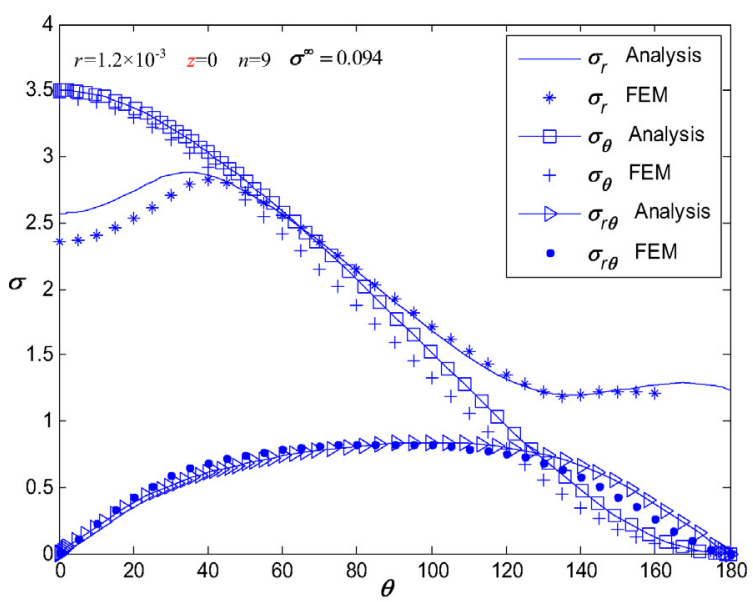

(c)

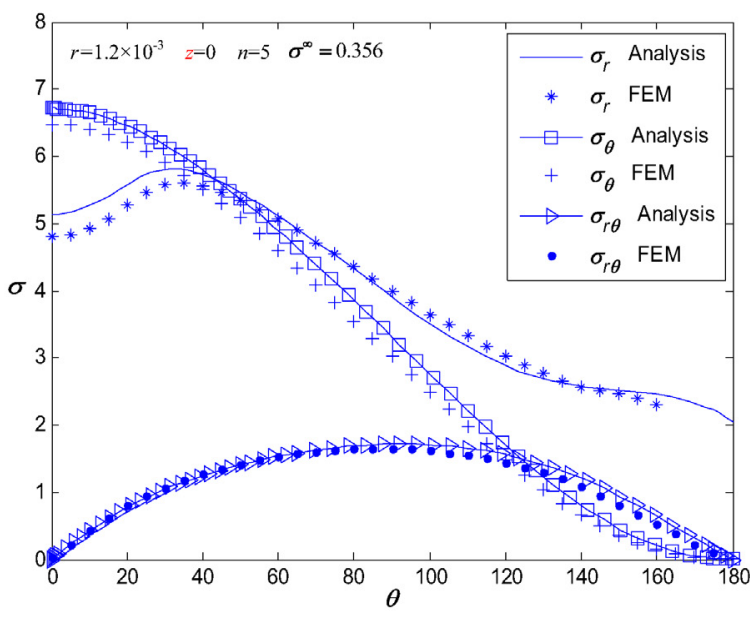

(b)

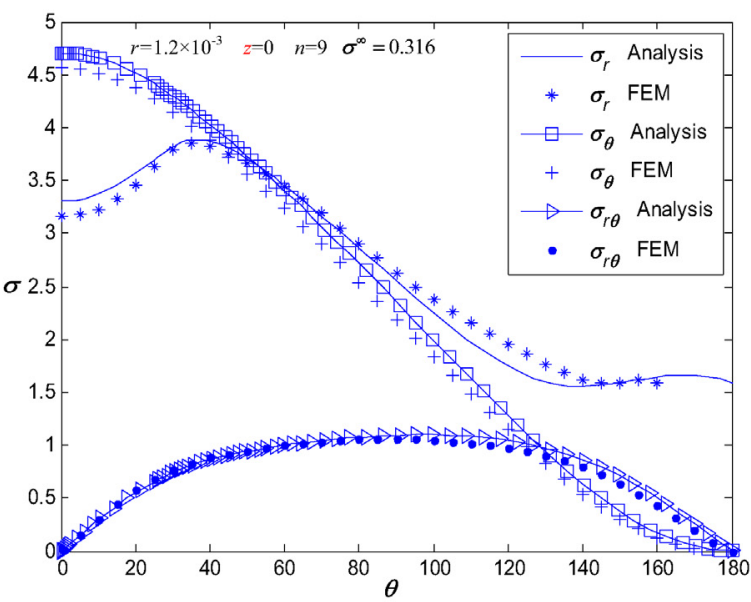

(d)

Fig. 11. $\theta$-variation of the stress fields near the crack front on the mid-plane. (a) $n=5, \sigma^{\infty}=0.211$, (b) $n=5, \sigma^{\infty}=0.356$, (c) $n=9, \sigma^{\infty}=0.094$, (d) $n=9, \sigma^{\infty}=0.316$

\section{Declaration of Competing Interest}

The authors declare that they have no known competing financial interests or personal relationships that could have appeared to influence the work reported in this paper.

\section{Acknowledgement}

The work done by Dr Liu Feng in Institute of Mechanics, Chinese Academy of Science is gratefully acknowledged.

\section{References}

[1] Rice JR, Rosengren GF. Plane strain deformation near a crack tip in a power-law hardening material. J Mech Phys Solids 1968;16:1-12. https://doi.org/10.1016/ 0022-5096(68)90013-6.

[2] Hutchinson JW. Singular behaviour at the end of a tensile crack in a hardening material. J Mech Phys Solids 1968;16:13-31. https://doi.org/10.1016/00225096(68)90014-8.

[3] Hartranft R, Sih GC. An approximate three-dimensional theory of plates with application to crack problems. Int J Engng Sci 1970;8:711-29. https://doi.org/10. 1016/0013-7944(75)90063-6.

[4] Sih GC. A review of the three-dimensional stress problem for a cracked plate. Int J Fract Mech 1971;7:39-61. https://doi.org/10.1007/BF00236482.

[5] Nakamura T, Parks DM. Three-dimensional stress field near the crack front of a thin elastic plate. J Appl Mech 1988;55:805-13. https://doi.org/10.1115/1. 3173725.

[6] Nakamura T, Parks DM. Three-dimensional stress field near the crack front of a thin ductile plate. J Mech Phys Solids 1990;38:787-812. https://doi.org/10. 1016/0022-5096(90)90040-b.

[7] Su XM, Sun CT. On singular stress at the crack tip of a thick plate under in-plane loading. Int J Fract 1996;82:237-52. https://doi.org/10.1016/10.1007/ 
bf00013160.

[8] Kwon SW, Sun CT. Characteristics of three-dimensional stress fields in plates with a through-the-thickness crack. Int J Fract 2000;104:289-314. https://doi.org/ 10.1023/a:1007601918058.

[9] Kotousov A. An application of the kane and mindlin theory to crack problems in plates of arbitrary thickness. Meccanica 2004;39:495-509. https://doi.org/10. 1007/s11012-004-5735-x.

[10] Codrington J, Kotousov A. The distributed dislocation technique for calculating plasticity-induced crack closure in plates of finite thickness. Int J Fract 2007;144:285-95. https://doi.org/10.1007/s10704-007-9100-7.

[11] Chaudhuri RA. Three-dimensional singular stress field at the front of a crack and lattice crack deviation (LCD) in a cubic single crystal plate. Phil Mag 2010;90:2049-113. https://doi.org/10.1080/14786430903571412.

[12] Sobotka JC, JrRH D. Steady crack growth in a thin, ductile plate under small-scale yielding conditions: Three-dimensional modeling. Engng Fract Mech 2011;78:343-63. https://doi.org/10.1016/j.engfracmech.2010.10.006.

[13] Khan SMA, Merah N, Adinoyi MJ. 3D effects on crack front core regions, stress intensity factors and crack initiation angles. Int J Solids Struct 2013;50:1449-59. https://doi.org/10.1016/j.ijsolstr.2013.01.019.

[14] Xiang M, Guo W. Formulation of the stress fields in power law solids ahead of three-dimensional tensile cracks. Int J Solids Struct 2013;50:3067-88. https://doi. org/10.1016/j.ijsolstr.2013.05.011.

[15] Kotousov A, Lazzarin P, Berto F, Pook LP. Three-dimensional stress states at crack tip induced by shear and anti-plane loading. Engng Fract Mech 2013;108:65-74. https://doi.org/10.1016/j.engfracmech.2013.04.010.

[16] Wang E, Zhou W, Shen G. Three-dimensional finite element analysis of crack-tip fields of clamped single-edge tension specimens-Part I: Crack-tip stress fields. Engng Fract Mech 2014;116:122-43. https://doi.org/10.1016/j.engfracmech.2013.10.022.

[17] Wang E, Zhou W, Shen G. Three-dimensional finite element analysis of crack-tip fields of clamped single-edge tension specimens-Part II: Crack-tip constraints. Engng Fract Mech 2014;116:144-57. https://doi.org/10.1016/j.engfracmech.2013.10.023.

[18] Shlyannikov VN, Boychenko NV, Tumanov AV, Fernández-Canteli A. The elastic and plastic constraint parameters for three-dimensional problems. Engng Fract Mech 2014;127:83-96. https://doi.org/10.1016/j.engfracmech.2014.05.015.

[19] Nikishkov GP, Matvienko YG. Elastic-plastic constraint parameter A for test specimens with thickness variation. Fatigue Fract Eng Mater Struct 2016;39:939-49. https://doi.org/10.1111/ffe.12390.

[20] Kotousov A, Khanna A, Bun S. Analytical evaluation of the transverse displacement at the tip of a semi-infinite crack in an elastic plate. Theor Appl Fract Mech 2018;93:288-92. https://doi.org/10.1016/j.tafmec.2017.09.011.

[21] Kishimoto K, Aoki S, Sakata M. On the path independent integral-J. Engng Fract Mech 1980;13:841-50. https://doi.org/10.1016/0013-7944(80)90015-6.

[22] Shih CF, Moran B, Nakamura T. Energy release rate along a three-dimensional crack front in a thermally stressed body. Int J Fract 1986;30:79-102. https://doi. org/10.1007/bf00034019.

[23] Carpenter WC, Read DT, Dodds Jr RH. Comparison of several path independent integrals including plasticity effects. Int J Fract 1986;31:303-23. https://doi.org/ $10.1007 /$ bf00044052.

[24] Chiarelli M, Frediani A. A computation of the three-dimensional J-integral for elastic materials with a view to applications in fracture mechanics. Engng Fract Mech 1993;44:763-88. https://doi.org/10.1016/0013-7944(93)90205-7.

[25] Rigby RH, Aliabadi MH. Decomposition of the mixed-mode J-integral—revisited. Int J Solids Struct 1998;35:2073-99. https://doi.org/10.1016/S0020-7683(97) 00171-6.

[26] Eriksson K. A general expression for an area integral of a point-wise J for a curved crack front. Int J Fract 2000;106:65-80. https://doi.org/10.1023/ a:1007646823223.

[27] Omer N, Yosibash Z. On the path independency of the point-wise J integral in three-dimensions. Int J Fract 2005;136:1-36. https://doi.org/10.1007/s10704005-3934-7. 